\title{
Energy-Dissipation Performance of Combined Low Yield Point Steel Plate Damper Based on Topology Optimization and Its Application in Structural Control
}

\author{
Haoxiang He, ${ }^{1,2}$ Xiaobing Wang, ${ }^{1}$ and Xiaofu Zhang ${ }^{1}$ \\ ${ }^{1}$ Beijing Key Laboratory of Earthquake Engineering and Structural Retrofit, Beijing University of Technology, Beijing 100124, China \\ ${ }^{2}$ Beijing Collaborative Innovation Center for Metropolitan Transportation, Beijing 100124, China \\ Correspondence should be addressed to Haoxiang He; hhx7856@163.com
}

Received 14 April 2016; Revised 31 May 2016; Accepted 1 June 2016

Academic Editor: Juan J. Del Coz Díaz

Copyright (C) 2016 Haoxiang He et al. This is an open access article distributed under the Creative Commons Attribution License, which permits unrestricted use, distribution, and reproduction in any medium, provided the original work is properly cited.

\begin{abstract}
In view of the disadvantages such as higher yield stress and inadequate adjustability, a combined low yield point steel plate damper involving low yield point steel plates and common steel plates is proposed. Three types of combined plate dampers with new hollow shapes are proposed, and the specific forms include interior hollow, boundary hollow, and ellipse hollow. The "maximum stiffness" and "full stress state" are used as the optimization objectives, and the topology optimization of different hollow forms by alternating optimization method is to obtain the optimal shape. Various combined steel plate dampers are calculated by finite element simulation, the results indicate that the initial stiffness of the boundary optimized damper and interior optimized damper is lager, the hysteresis curves are full, and there is no stress concentration. These two types of optimization models made in different materials rations are studied by numerical simulation, and the adjustability of yield stress of these combined dampers is verified. The nonlinear dynamic responses, seismic capacity, and damping effect of steel frame structures with different combined dampers are analyzed. The results show that the boundary optimized damper has better energy-dissipation capacity and is suitable for engineering application.
\end{abstract}

\section{Introduction}

In order to resist the dynamic action of strong earthquakes and hurricanes, building structures should have enough capacities to dissipate energy and avoid severe damage. The seismic performance of the traditional buildings is improved by enhancing the mechanical parameter such as strength, stiffness, and ductility; that is, the structure can reserve and dissipate the input energy by self-resistance. Therefore, the structure designed by normal methods does not have the capability of self-adjustment, which may lead to unacceptable damage and even collapse during earthquakes and the safety requirements are hard to meet. Hence, the traditional seismic design methods need to be improved by inducing new energy-dissipation technology. Structural control provides a safe and effective way to enhance the aseismic capacity of structures. Structural seismic control is to change or adjust the dynamic characteristic or dynamic action by installing devices (such as seismic isolation bearing), some mechanisms (such as energy-dissipation braces and joints, fluid viscous damper, and metallic damper), some substructure (such as tuned mass damper), or external force (such as external energy input) in a certain part of structure. Under small earthquake and wind, the structure itself has enough lateral resistance to meet the operating requirement, and structure is in the elastic state. Under severe earthquake and strong wind, the lateral deformation of the structure will continuously increase, and the energy-dissipation devices firstly enter the inelastic state, provide enough damping, dissipate the vibration energy, and rapidly attenuate the vibration responses of the main body structure so as to reduce the damage degree. The realization of structural seismic control mainly depends on the application of simple and effective dampers and energy-dissipation devices. In recent years, a large number of dampers, such as mild steel damper, friction damper, viscous fluid damper, and smart damper, are developed and 
utilized in practical engineering, and the damping effect is very obvious.

Low yield point metal damper is a kind of passive energydissipation device, which has a wide range of applications, and the advantages include simple conformation, stable hysteretic performance, low cost, and explicit mechanism. By using plastic hysteresis deformation of different forms of metals to dissipate energy, low yield point metal damper has superior hysteretic characteristics during plastic stage and absorbs a large amount of energy in the process of elastic-plastic hysteresis. Thus, it is used as energy-dissipation dampers in civil engineering with diverse types [1]. Since Kelly et al. [2] put forward the concept of energy dissipation with metal energy-dissipation devices and carried out the relevant experimental study in 1972, many theoretical and experimental researches on metal dampers are carried out. Various forms of low yield point metal dampers are studied, such as U-shaped steel plate damper, conical steel damper, shear yielding type steel damper, and axial yield damper. Kajima Company proposed a honeycomb metallic yield damper, which can be installed in walls or beams. Whittaker et al. [3] and Tsai et al. [4] firstly proposed displacement bending type of dampers with $\mathrm{X}$ shape and triangle shape. Tirca et al. [5] proposed a kind of steel damper which only generates in-plane deformation and the corresponding highrise structure with such dampers was analyzed, and it is verified that the dampers have excellent energy-dissipation capacity. Zhou and Liu [6, 7] developed several new metal energy-dissipation devices, such as the circular ring damper and double circular ring mild steel damper. Zhang et al. [8] presented a mild steel damper with a diamond shape hollow in-plane, and this kind of damper has the advantages of large plastic deformation and full hysteresis curve, but its initial stiffness is small and the total amount of steel is large. In order to improve the initial stiffness of dampers, Mito et al. [9] put forward a shear panel damper with rectangle shape, but the plane stress of the four corners on the plate can prematurely concentrate and the energy dissipation capacity is inadequate, and G. Li and H. Li [10] proposed the mild steel dampers with a single circular hole and double $\mathrm{X}$ shape. The initial stiffness of these dampers is relatively large and hysteretic curve is full, but there are still some disadvantages such as obvious stress concentration, limited yield area, and low utilization ratio.

Although the research and application on energydissipation technology of low yield point steel dampers have achieved great progress, there are still many problems to be studied and solved: (1) the existing low yield point steel dampers are usually packaged structures, which are not convenient for installation and adjustment, and the maintenance cost is high. (2) The damping performance of the dampers cannot be fully embodied in the small and medium earthquakes but only works in strong earthquakes and hurricanes due to the high yield strength; thus, the ultralow-yield point dampers are highly required especially for low and medium level vibration. In order to maximize the effect of the energy dissipation, the damper should have the capability of both large initial stiffness and good deformation energy-dissipation capacity after yield. (3) The adjustability of the current dampers is deficient, and the research on seismic strengthening and repair of existing building structures with combined low yield point steel dampers should be enhanced. (4) The resilient function and replaceability of the low yield point steel damper are insufficient, and it needs to develop new type of damper to fulfill the requirements of earthquake resilient structure. Therefore, it is significant to develop novel dampers with low cost and low yield strength. In view of this, a combined low yield point steel plate damper is presented in this study, the yield stress is low, and it can be adjusted according to engineering demand. At the same time, the "maximum stiffness" and "full stress state" are both used as optimization objectives to achieve the topology optimization of damper shape in order to make the damper have ideal deformation capacity and energy-dissipation capacity. During an earthquake, low yield materials firstly deform and reach the yield stage to fully dissipate the energy, and there is no serious plastic deformation in the original main structure, so the overall seismic performance and safety are guaranteed. After the main earthquake, the combined steel plate with low yield point material can be quickly replaced to restore the global seismic performance, which makes the main elements and frame structure have the ability to resist the aftershocks.

\section{Composition and Characteristics of Composite Low Yield Point Steel Damper}

2.1. Material Property Test of Low Yield Point Steel. The material in the low yield point damper is different from the normal steel in traditional steel structures, its yield strength is low, plastic deformation may occur under small strain, it has sufficient ductility and bearing capacity, and the low cycle fatigue performance is superior. Hence, one of the key technologies of low yield point steel damper production is to select the metal with lower yield strength and larger extensibility. For the conventional low yield point metal including mild steel or low yield point steel, lead, aluminum, and zinc-aluminum alloy the common characteristics of these materials are that the plastic deformation capability is strong, the low cycle fatigue property is superior, and the hysteretic performance is stable and can be recycled. At present, there are many theoretical studies on the shock absorption of low yield point metal, but the studies on the steel product which has high stability and safety and simultaneously meets the requirements of engineering applications are still rare [11].

In this study, the new developed low yield point steel LY160 (yield stress is about $160 \mathrm{MPa}$ ), which is made in China, is selected as the energy-dissipation material and the corresponding experimental study is carried out. In order to achieve low yield point performance, the original material of LY160 mainly consists of the composition of industrial pure iron, and a small amount of $\mathrm{Ti}$ and $\mathrm{Al}$ is added to retain carbon and nitrogen atoms, so as to reduce the hindrance of the dislocation motion. In addition, the rolling technique is adjusted to realize grain growth coursing and obtain the single ferrite organization. Finally, the special steel has excellent plasticity, low yield strength ratio, and good low cycle fatigue performance. In order to obtain and compare the performance parameters of different steel materials, 


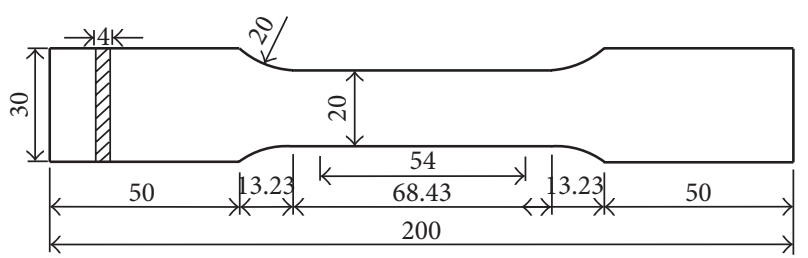

FIGURE 1: Diagram of specimen size (unit: $\mathrm{mm}$ ).

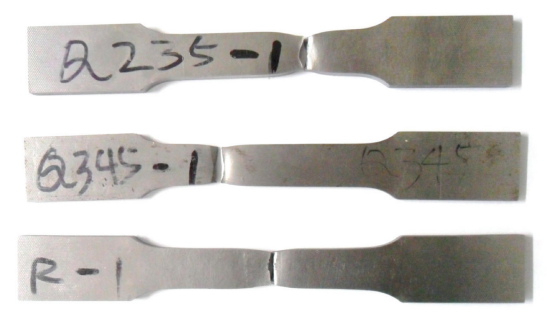

FIgURE 2: Specimens with tensile fracture.

the comparative research on Q345 steel (yield point is about $345 \mathrm{MPa}$ ), Q235 steel (yield point is about $235 \mathrm{MPa}$ ), and LY160 steel (yield stress is about $160 \mathrm{MPa}$ ) is carried out. The specimens are sheet tensile components, and they are processed in accordance with the relevant provisions of guidelines [12]. The diagram of specimen size is shown in Figure 1.

Three specimens made in each kind of material are produced and different types of specimens are shown in Figure 2. The quasistatic tensile test of the specimens is performed on a normal stretching testing machine, as shown in Figure 3. The longitudinal extensometer is placed on the specimens to measure the longitudinal strain, and the stretching rate is $3 \mathrm{~mm} / \mathrm{min}$. The experimental results are shown in Figure 4 and Table 1. It is evident that the low yield point steel has expected yield stress and super ductility. According to the comparison between the yield strength and the nominal yield strength in Table 1 , it can be seen that the yield point of LY160 is relatively stable, and the difference between the yield point and the nominal value is about $2 \%$. In summary, LY160 steel has the ideal low yield point, excellent ductility, and toughness to dissipate energy, so it can be used as the material of the low yield point damper.

\subsection{Composition of Combined Low Yield Point Steel Plate} Damper. In view of the limitation of the yield point of the traditional metal damper, a new type of combined low yield point steel plate damper is proposed. This damper includes the upper and lower horizontal connecting steel plates connected with the structure and the metal plates with the hollow parts arranged between upper and lower horizontal connecting plate. The metal plates are composed of low yield point steel plate (such as Q160) and ordinary steel plate (such as Q345) with different combined ratio, and the thickness is the same, so the equivalent yield strength is lower and can be adjusted or controlled. Two types of metal plates are arranged

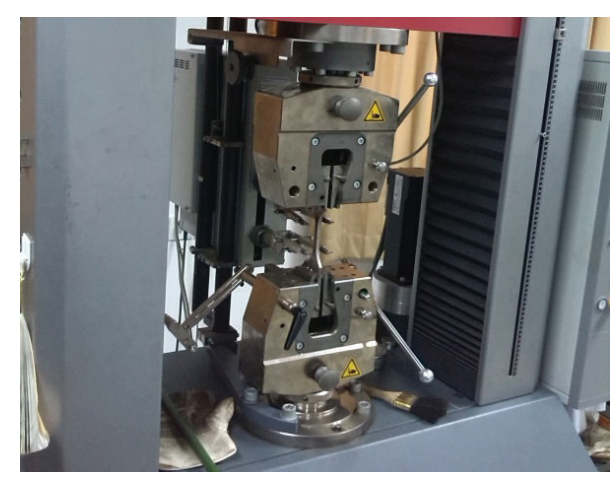

FIGURE 3: Stretching testing machine.

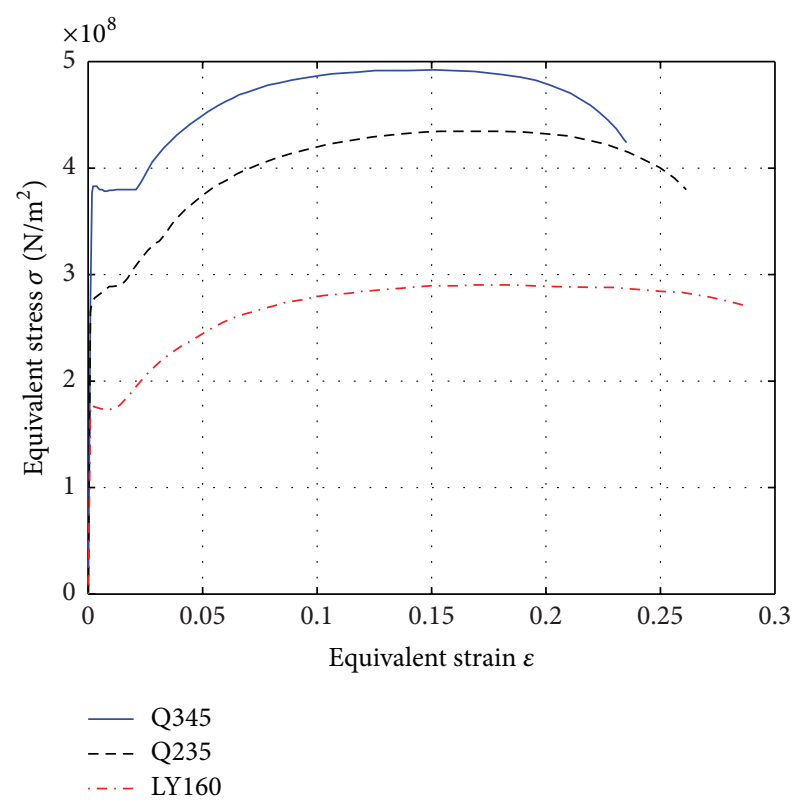

FIGURE 4: Stress-strain curve of different steels.

alternately between the fixed connections and connected by high strength bolts, and the specific construction detail is shown in Figure 5. The number, size, and hollow form of the energy-dissipation metal plates should be determined based on the anticipated yield strength and fixed effect between metal plates, so as to ensure adequate deformation capacity.

The combined low yield point steel plate damper can be installed in the structure beams or the infill walls with the cooperation of braces. During earthquakes, the damper firstly enters the plastic state to dissipate the earthquake energy and ensure the safety of the main structure.

Compared with other steel shear dampers, the advantages of this novel damper are as follows: (1) the energy-dissipation steel plates with different yield points are assembled, so the yield stress of this damper is lower, and the combined steels can fully work in small and moderate earthquakes, and the hysteretic behavior is fulfilling. (2) Compared with the damper only using Q160 steel, the combined damper has better controllability, the best damping performance and energy-dissipation effect can be obtained by modulating 
TABLE 1: Results of monotonic tensile test.

\begin{tabular}{|c|c|c|c|c|c|}
\hline Strength grade & Sample number & Yield stress (MPa) & Tensile stress (MPa) & Elongation (\%) & $\begin{array}{c}\text { Difference between yield point } \\
\text { and nominal value (\%) }\end{array}$ \\
\hline \multirow{4}{*}{ Q345 } & Number 1 & 387.2 & 496.6 & 22.2 & 12.2 \\
\hline & Number 2 & 383.3 & 485.5 & 24.4 & 11.1 \\
\hline & Number 3 & 385.5 & 492.6 & 23.5 & 11.7 \\
\hline & Mean & 385.3 & 491.6 & 23.4 & 11.7 \\
\hline \multirow{4}{*}{ Q235 } & Number 1 & 279.2 & 435.5 & 30.4 & 18.8 \\
\hline & Number 2 & 276.5 & 433.0 & 26.3 & 17.7 \\
\hline & Number 3 & 273.6 & 434.5 & 25.4 & 16.4 \\
\hline & Mean & 276.4 & 434.3 & 27.4 & 17.6 \\
\hline \multirow{4}{*}{ LY160 } & Number 1 & 160.2 & 262.2 & 30.9 & 0.0 \\
\hline & Number 2 & 165.1 & 266.1 & 33.4 & 3.2 \\
\hline & Number 3 & 164.5 & 260.4 & 30.6 & 2.8 \\
\hline & Mean & 163.3 & 262.9 & 31.6 & 2.0 \\
\hline
\end{tabular}

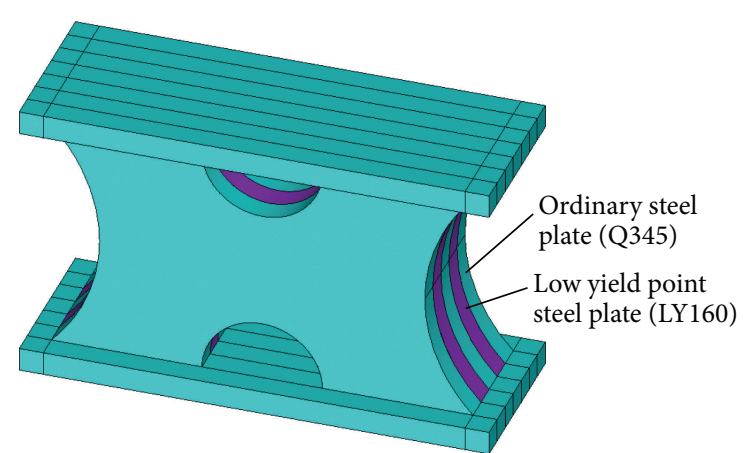

Figure 5: Composition of combined low yield point steel plate damper.

the thickness ratio of two types of steel plates, and it is convenient to install, upgrade, and maintain. (3) The construction of this damper is simple and the materials are low cost, so it has wide prospect in application.

\section{Double Topology Optimization Design of Damper}

3.1. Shape Optimization Design of Damper. The normal low yield point steel plate damper consists of rectangular steel plates or local hollow steel plates. The rectangular steel plates usually just yield in the limited area of central part, so the material utilization ratio is low and the global deformation is small. The local hollow plate can yield at each point of the same thickness along the direction of length side, which will significantly improve the energy-dissipation capacity and deformation capacity [13]. In addition, some low yield point plate dampers resist the external forces in the form of outof-plane style, and the energy dissipation is realized through the obvious plastic deformation after the plates yield due to bending. However, the initial stiffness and the load capacity of the damper are both small. If the steel plates dissipate energy by shear deformation in the plane, the damper will have a larger initial stiffness but the damage due to stress concentration or local buckling is prone to occur. Hence, deformation capacity and energy-dissipation capacity of the damper may not reach the expected requirement if there are no optimizations for the hollow form of steel plates.

In view of the above problems, Deng et al. [14] used the finite element analysis to simulate the traditional shear type steel plate damper and obtained the optimal shapes of plates with various sizes, as shown in Figure 6(a). After the shape optimization, the low cycle fatigue capacity of shear type steel plate damper is significantly improved, but the improvement of the dissipation capacity is not obvious. Wang and An [15] presented a new steel plate damper, as shown in Figure 6(b). The initial stiffness and the yield stress are both larger, the stress distribution is uniform, and no obvious stress concentration occurs under cyclic loading, but it is easy to appear as out-of-plane buckling. G. Li and H. Li [10] presented a double $\mathrm{X}$ type steel damper by changing the geometry of the steel plates, as shown in Figure 6(c). The multiple point yields will occur in the plates, and better energy-dissipation effect can be achieved, but the stress concentration is obvious and the yield area is small; the utilization ratio of steel is not high enough.

The main reason for the shortage of the above steel plate dampers is that the determination of the hollow forms mainly depends on engineering experience, but the explicit optimization objectives and solid theoretical support are not adequately studied, so the corresponding hollow forms are not optimal. Therefore, it is necessary to carry out a comprehensive optimization for external shape and hollow form under the guidance of the topology optimization theory.

Structural topology optimization mainly refers to the optimization of structural form, also known as the shape optimization. The goal of topology optimization is to find the optimal material distribution scheme of the structure under the given design domain, constraint condition, and loading. In the traditional topology optimization method, the optimization criterion method is generally used as the basic solution method, and the "maximum stiffness" is selected as 


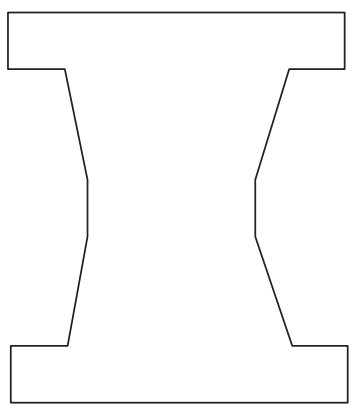

(a) Shear type damper

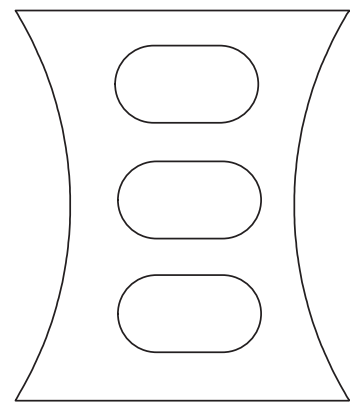

(b) Yield energy-dissipation damper

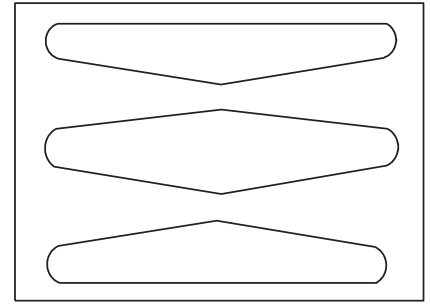

(c) Double X type damper

Figure 6: The hollow form of steel plate.

the optimization objective to obtain the maximum stiffness under the designated volume constraint. However, only the structural elastic analysis can be carried out in the process of the optimization for "maximum stiffness," and the stress state of the material is not fully considered, so the stress distribution is not uniform even in the optimal solution. Hence, the actual energy-dissipation capacity of the damper scheme obtained by the above topology optimization method may not be optimal, and it is significant to realize comprehensive optimization by combining other optimization objectives [16-21].

As a kind of basic structural optimization design method, full stress design is widely accepted and used in the structural optimization analysis. The stress of most parts of the material can reach the allowable value under designated load by the full stress design method, so the material is fully utilized and the full stress state can be used as the optimization objective of the structural topology optimization design. The full stress design method can solve the problem of elastic-plastic analysis, but the disadvantage is that it is usually applied to the optimal design of statically determinate structure under static load, so it is not a perfect method also [22-24]. In conclusion, if "maximum stiffness" and "full stress state" are both used as the optimization objectives, after a number of iterations and modifications, the performance of the material can be fully utilized under the condition of large stiffness and the optimum balance of stiffness and full stress can be achieved, so that a more comprehensive optimization scheme can be obtained. Therefore, a topology optimization method based on full stress is proposed and applied to the shape optimization of steel plate in order to enable the low yield point steel plate damper that has the optimal hysteretic behavior and energy-dissipation capacity.

For general topology optimization analysis software, the "maximum stiffness" and "full stress state" can be used as the optimization objective alternately, and the double optimization results for specified volume reduction ratio are obtained, and then the optimization results of different volume reduction rates are compared to determine the final optimization scheme. The specific optimization process is shown in Figure 7.
3.2. Optimization Model and Results. In this study, shape optimization of the combined low yield point steel plate is executed according to the design flow of shape topology optimization and optimization philosophy based on the full stress. Based on the double $\mathrm{X}$ model proposed in paper [10] and the deficiency of its shear capacity, an interior optimized damper form is presented, as shown in Figure 8(a). In addition, since the material utilization rate of the unilateral optimization model in paper [14] is not the maximum, a boundary optimized damper model is presented, as shown in Figure 8(b). Furthermore, the model proposed in paper [15] is improved and the ellipse optimized model is proposed to overcome the deficiency of easy buckling out of the plane, as shown in Figure 8(c). The double optimization on the initial shape of three types of dampers in Figure 8 is carried out. The length of each model is $350 \mathrm{~mm}$, the width is $200 \mathrm{~mm}$, and the thickness is $20 \mathrm{~cm}$.

At first, the finite element model of the interior optimized damper is established, as shown in Figure 8(a), and the bottom of the model is fixed and horizontal load is applied on the top end. Secondly, the model shape topology optimization design for the specified volume reduction rate is carried out, and the final stress nephogram is obtained, as shown in Figure 9(a). If the maximum stress distribution is uniform or the colors of the stress nephogram are close, it can be considered that the model is close to the full stress state and the current model is determined as the final optimization scheme for the specified volume reduction rate. Otherwise, the region or the volume of lowest stress should be weakened appropriately and the damper model should be modified, as shown in Figure 9(b). Then the topology optimization is carried out again, until the stress distribution of the model reaches full stress state, namely, the optimal size is obtained for this volume reduction ratio, as shown in Figure 9(c). Finally, the optimization results and the overall effect of the model under various volume reduction conditions are generally considered, and the final optimization scheme is determined, as shown in Figure 9(d).

According to the flow chart in Figure 5 and the double optimization process of interior optimized damper, the final scheme of the boundary optimized damper model can be 


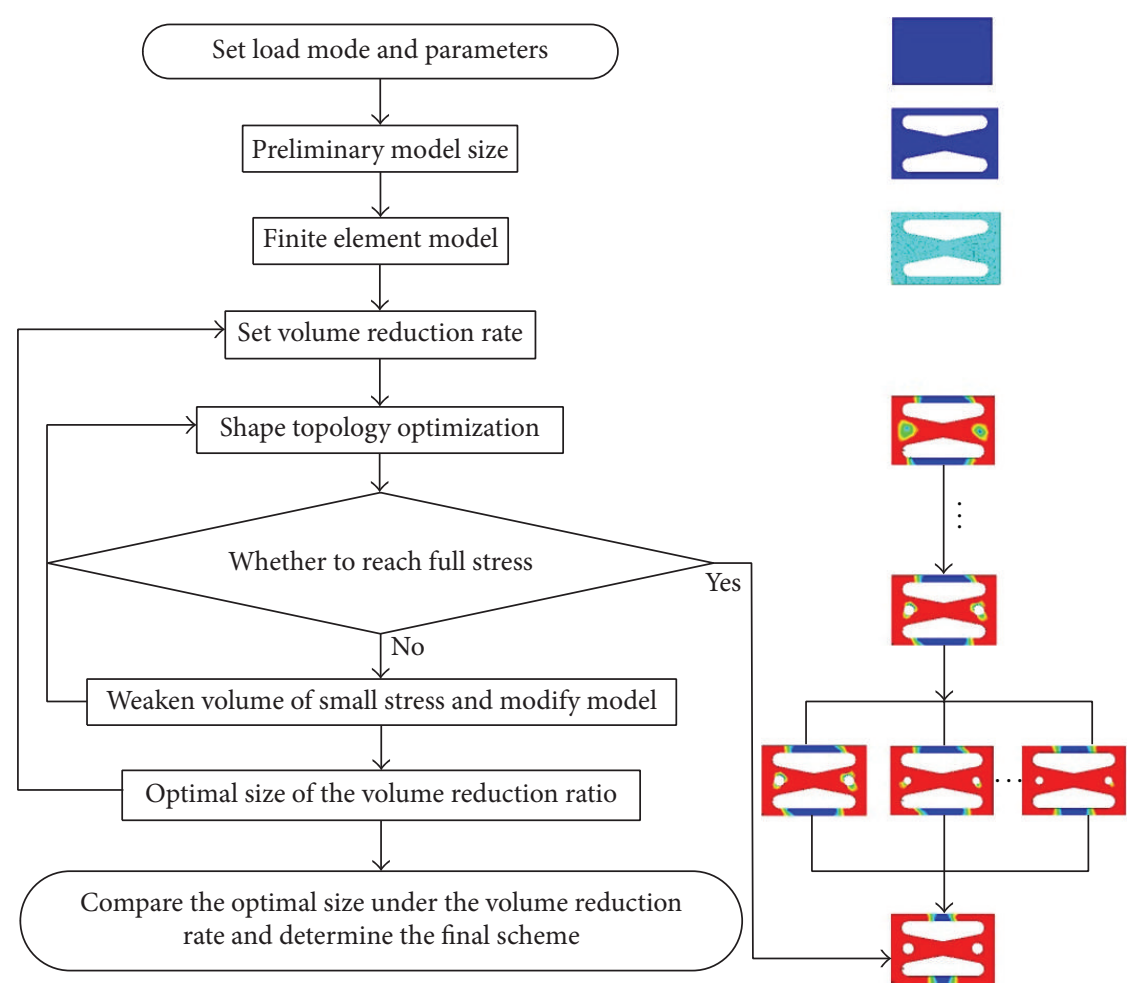

FIgURE 7: Optimization process.

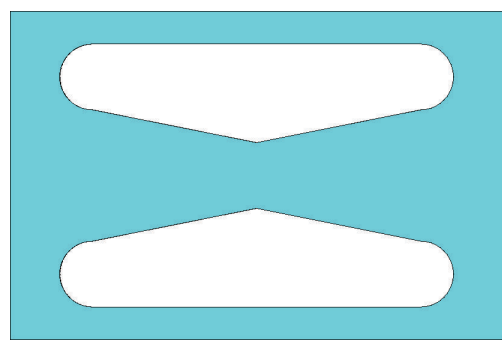

(a) Interior hollows

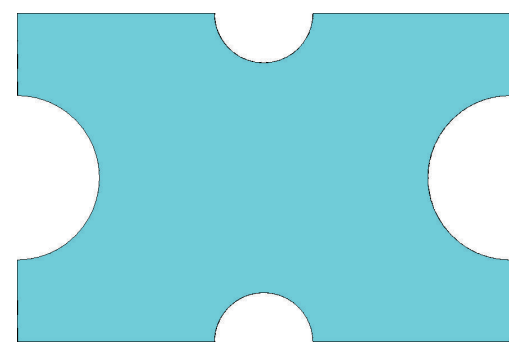

(b) Boundary hollows

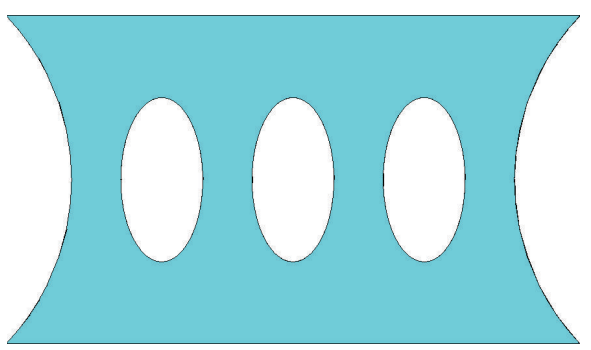

(c) Ellipse hollows

Figure 8: The initial form of damper.

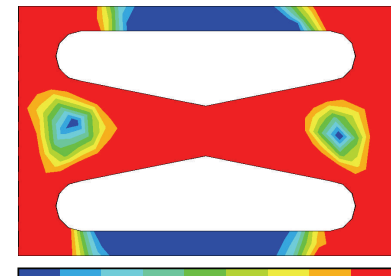

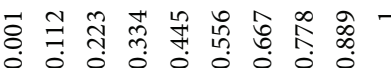

(a) Preliminary optimization

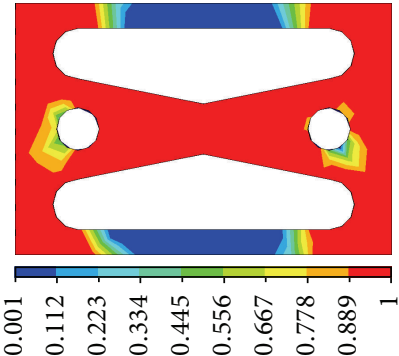

(b) Weakening scheme

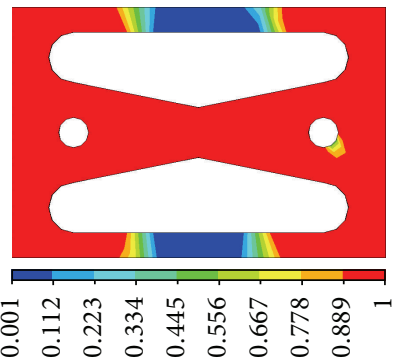

(c) Optimization scheme of full stress state

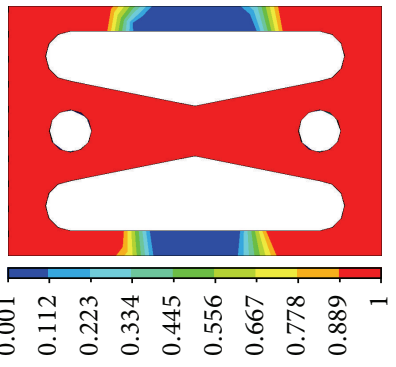

(d) Final optimization scheme

FIgURE 9: Optimization process of interior optimized model. 


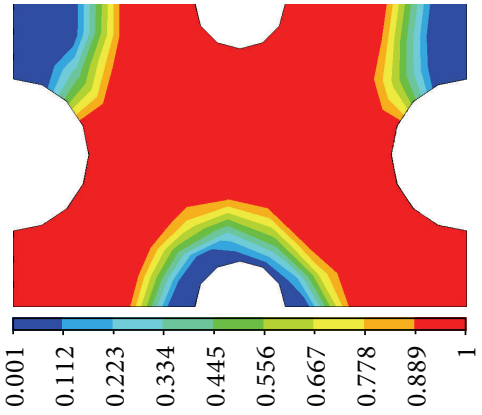

(a) Preliminary optimization

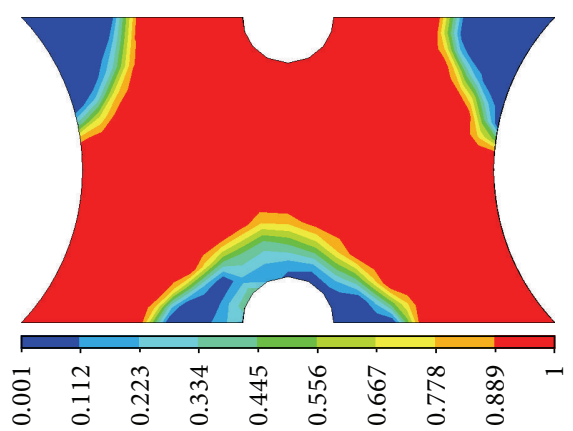

(b) Optimal size of weakening small stress

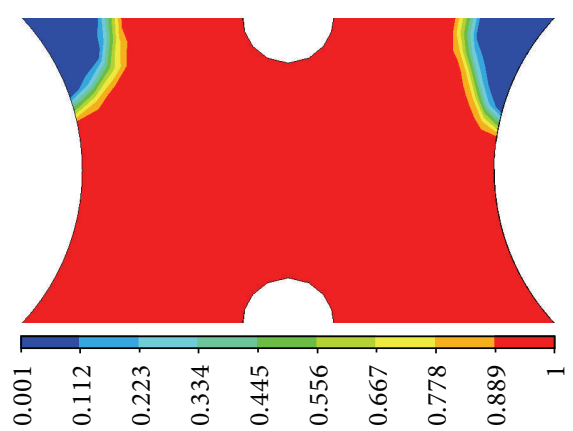

(c) Final optimal scheme

FIGURE 10: Optimization process of boundary optimized model.

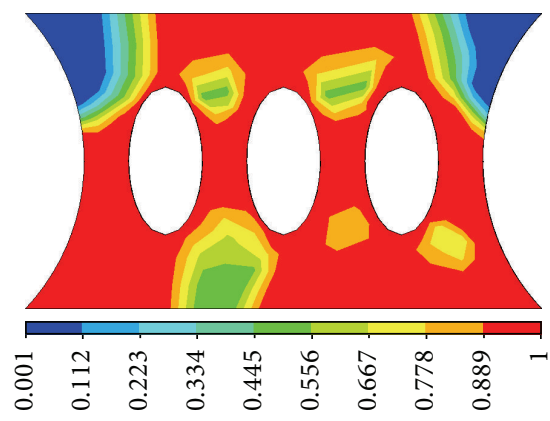

(a) Preliminary optimization

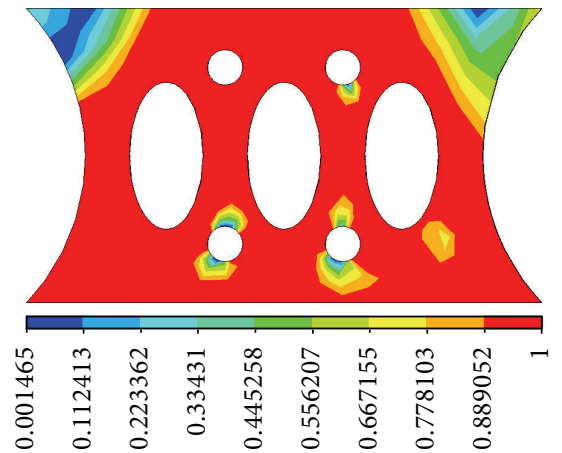

(b) Optimal size of weakening small stress

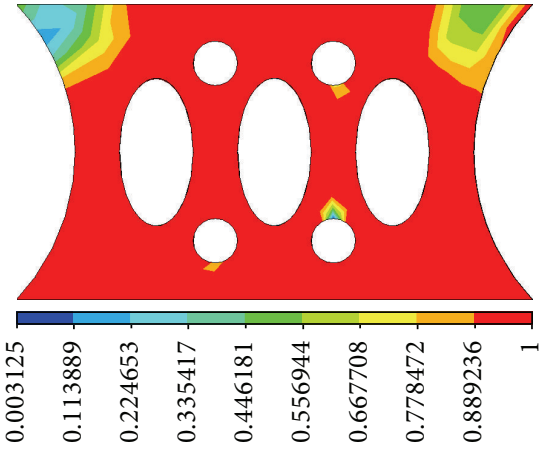

(c) Final optimal scheme

FIGURE 11: Optimization process of ellipse optimized model.

obtained, as shown in Figure 10. In addition, the optimal result of the ellipse optimized model is shown in Figure 11. The final dimension charts of all the optimized dampers are shown in Figure 12.

\section{Performance Simulation of Combined Dampers}

4.1. Performance Comparison of Different Shape Dampers. After the optimal forms of these dampers are determined, it is necessary to carry out a more intensive elastic-plastic analysis to further verify the energy-dissipation performance of the dampers. The finite element models of different types of combined low yield point steel plate dampers are established, as shown in Figure 5. The first kind of plate is made in Q345 steel, and the second kind of plate is made in LY160 steel. The top end of the model is fixed, and the low cyclic loading is applied to the bottom. The loading mode is variable amplitude loading according to displacement, as shown in Figure 13. The overall hysteretic curves of the three models are shown in Figure 14. The stress nephograms of Q345 steel plate and Q160 steel plate with different forms are shown in Figures 15 and 16, respectively.

It can be seen from the simulation results and hysteresis curves that the yield shearing force of the interior optimized damper is smaller and the deformability is outstanding.
Furthermore, the hysteretic curve of the boundary optimized damper model is fullest, and the initial stiffness is maximum, so this model has excellent energy-dissipation capacity.

It can be seen from the stress nephograms that the stress distribution of the interior optimized damper is relatively homogeneous but the stress level is rather low. On the contrary, the stress level of boundary optimized damper is high and the stress distribution is also homogeneous, so the full stress design criteria are ideally realized. In addition, the stress concentration of the ellipse optimized model is obvious, and the utilization rate of material is lowest.

The skeleton curves of different combined low yield point steel plate dampers under cyclic loading are shown in Figure 17. Three kinds of dampers are in the elastic stage before the displacement is less than $0.05 \mathrm{~mm}$. After the displacement increases to $0.1 \mathrm{~mm}$, all the dampers entered into the yield stage. Under the action of the same force, the displacement of the boundary optimized damper is minimum, and its initial stiffness is significantly larger than that of the other two dampers. It is obvious that the ductility and the aseismic capability of the boundary optimized damper is superior and the bearing capacity and the initial stiffness of main structure can be effectively improved if this damper is applied.

In conclusion, the interior optimized damper is suitable for the engineering structures such as large-span spatial structure and workshop structures, which needs the dampers to resist large deformation. Because the energy-dissipation 


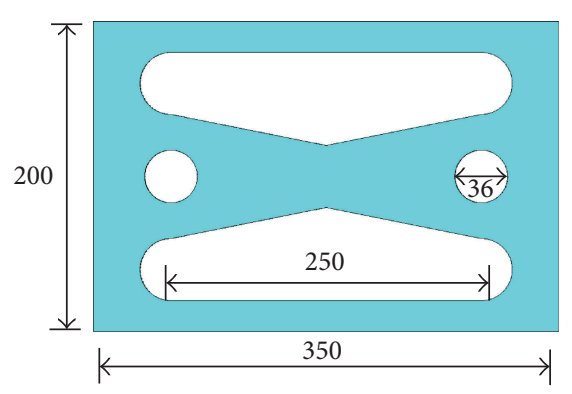

(a) Interior optimized model

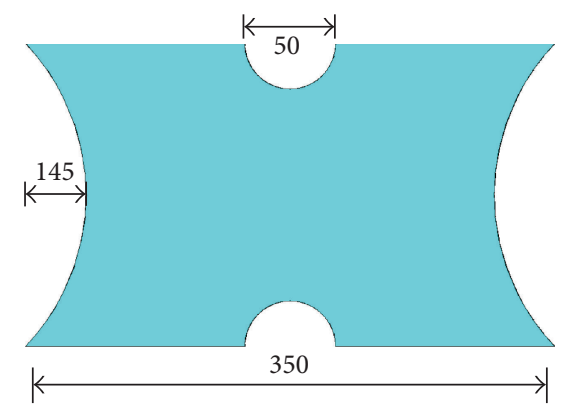

(b) Boundary optimized model

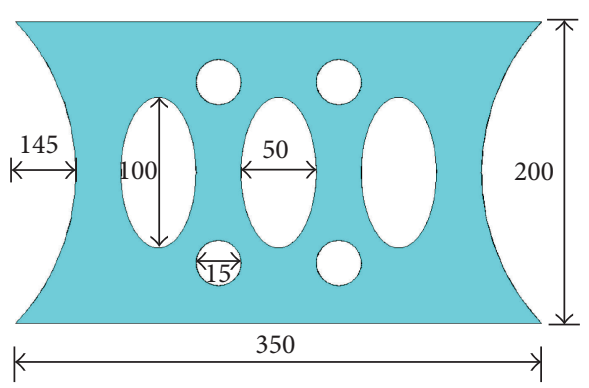

(c) Ellipse optimized model

FIGURE 12: Final optimized size (unit: mm).

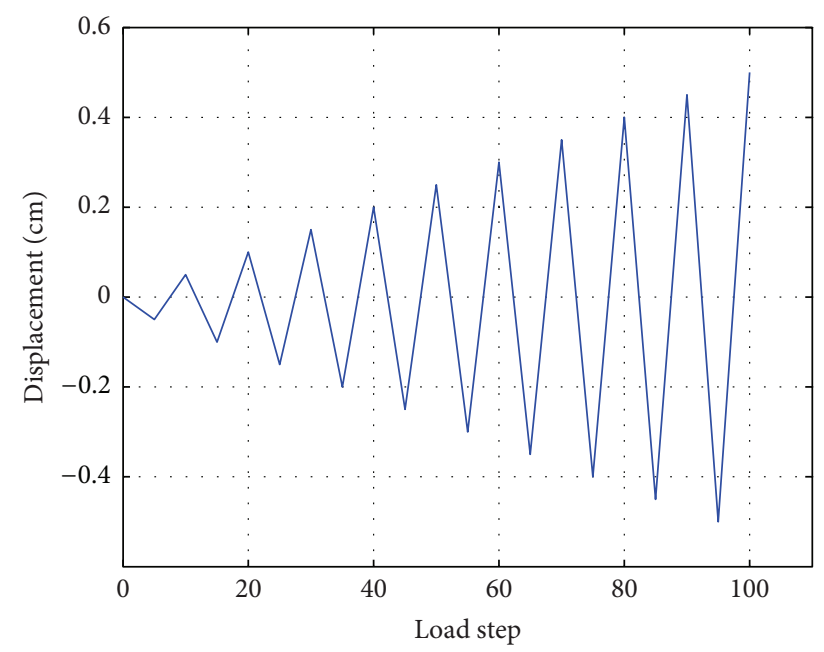

FIGURE 13: Loading mode.

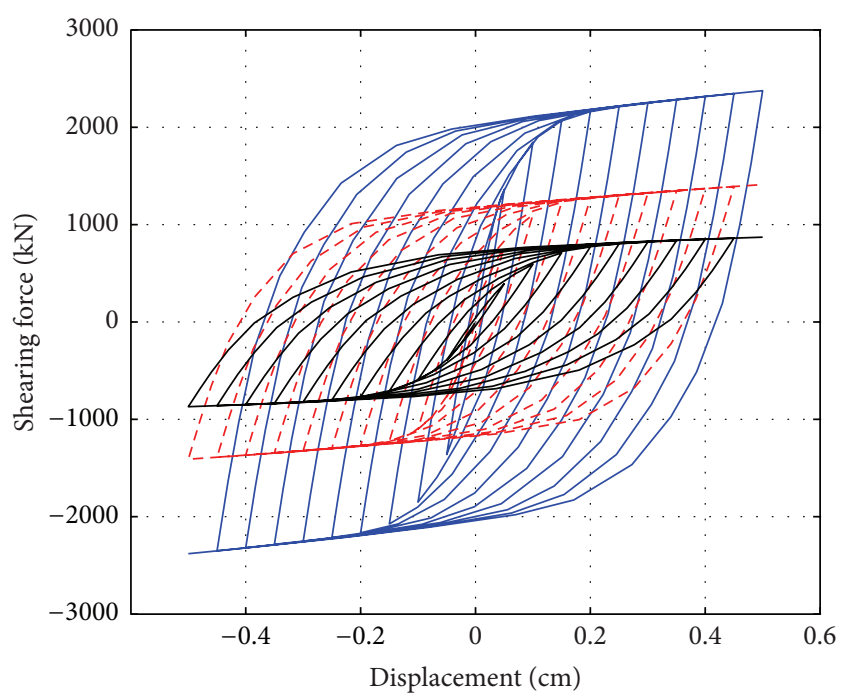

_- Boundary optimized damper
- - Ellipse optimized damper
- Interior optimized damper

FIGURE 14: Comparison of hysteresis curves. capacity of the boundary optimized damper is strong and the comprehensive performance is optimal, it is applicable for common structures. Since the ellipse optimized damper may lead to device failure due to stress concentration, it is not recommended for normal structures.

To compare the energy-dissipation capacity of different types of dampers in more explicit parameters, Park-Ang damage index [25] and the equivalent hysteretic damping ratio calculation model are used to evaluate the performance under low cyclic loading.

As a classic damage index, Park-Ang index can be calculated as follows:

$$
\mathrm{DI}=\frac{x_{m}}{x_{c u}}+\beta \frac{E_{h}}{F_{y} x_{c u}},
$$

where $x_{c u}$ is the limit displacement under monotonic loading; $F_{y}$ is the yield strength; $x_{m}$ and $E_{h}$ are the actual maximum deformation and cumulative hysteretic energy dissipation, respectively. $\beta$ is the energy-dissipation factor of component, and the value is set as 0.15 in this study.

The equivalent hysteretic damping ratio $\xi$ can be calculated as follows $[26,27]$ :

$$
\xi=\frac{E_{D}}{4 \pi E_{S}},
$$

where $E_{D}$ is the hysteretic dissipated energy under structural single cycle motion, which is equal to the envelope area of the hysteresis loop, and $E_{S}$ is maximum strain energy.

The Park-Ang damage index curves and the equivalent hysteretic damping ratios of each damper under different displacement are calculated, as shown in Figures 18 and 19, respectively. It can be seen that the energy-dissipation capacity of each damper is close at the initial stage, and the energydissipation capacity gradually enlarges with the increase of deformation. At the later stage of loading, the equivalent damping ratio of the boundary optimized damper and the ellipse optimized model grows faster, which indicates that the corresponding energy-dissipation capacity rises rapidly, and the growth rate of the energy-dissipation capacity of the interior optimized damper is slightly lower.

4.2. Adjustability Verification of Combined Dampers. From the above analysis, it is assumed that the boundary optimized 


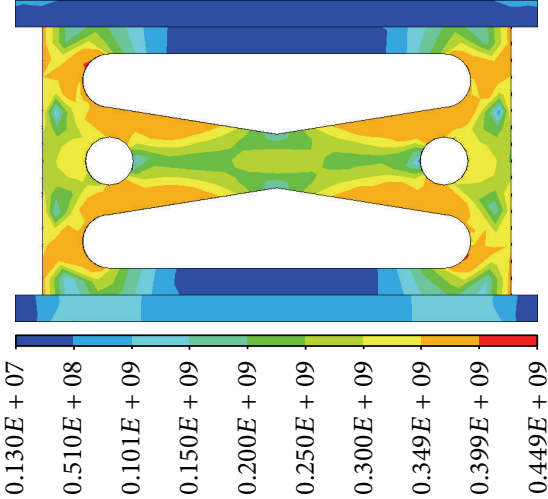

(a) Interior optimized model

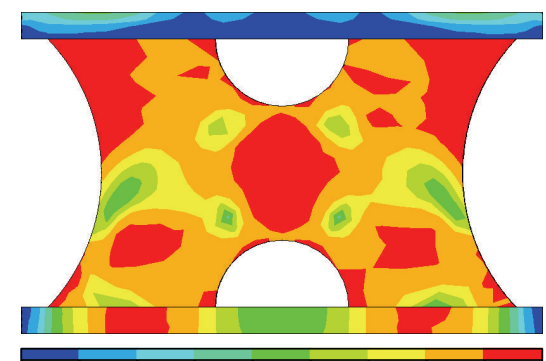

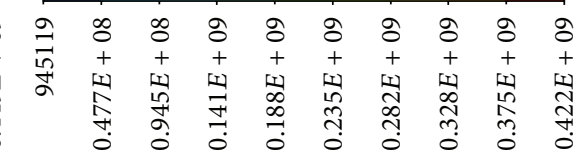

(b) Boundary optimized model

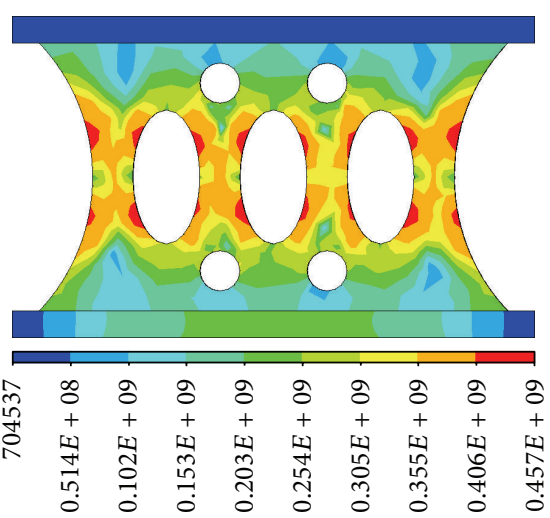

(c) Ellipse optimized model

FIGURE 15: Stress nephogram of Q345 steel plate.

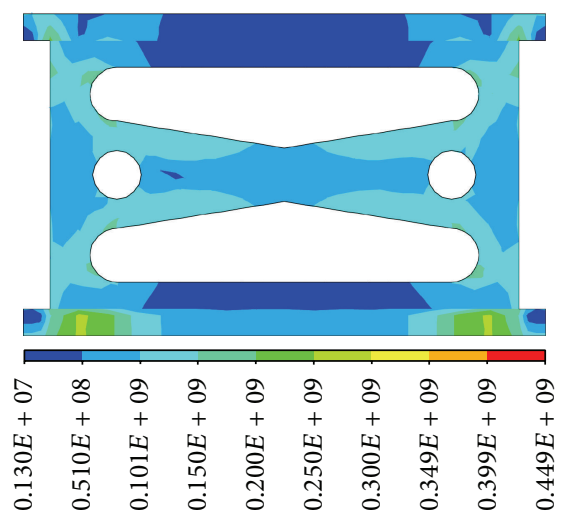

(a) Interior optimized model

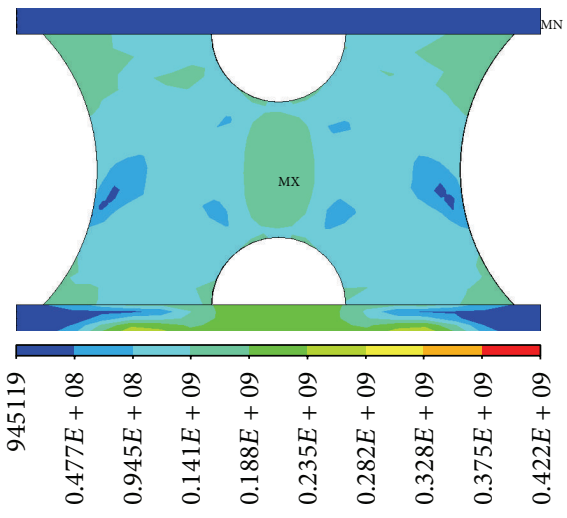

(b) Boundary optimized model

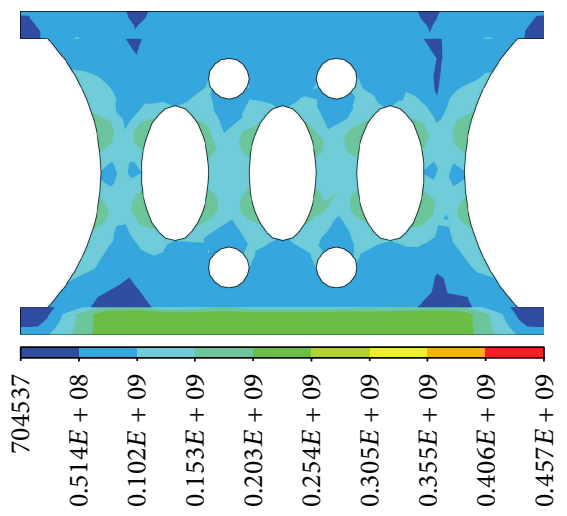

(c) Ellipse optimized model

FIGURE 16: Stress nephogram of LY160 steel plate.

damper and the interior optimized damper have excellent energy-dissipation capacity and stability. In order to verify the adjustability of these combined dampers, the effect of different materials ratio on the performance of the damper is studied.

Three schemes are designed to analyze the performance of the combined dampers, and 5 steel plates with the same thickness are adopted to assemble the dampers; only the material properties and the proportion of steel plates are different. For the first scheme, all the 5 steel plates are made in LY160, and Q345 steel plates are used for the second scheme. In the third scheme, the damper is alternatively assembled by 3 pieces of Q345 plates and 2 pieces of LY160 plates. The finite element software is used to simulate the damper models, and the dampers are applied to low cyclic loading. The hysteresis curves of the interior optimized damper and the boundary optimized damper is shown in Figures 20 and 21 , respectively. From the comparison of the hysteresis curves, it is evident that the yield stress of the third scheme is between the corresponding values of the first scheme and the second scheme. Hence, the yield stresses of the combined dampers can be adjusted by changing the combination proportion of different materials.

\section{Energy-Dissipation Effect of Combined Dampers on Frame Structure}

The characteristics such as large initial stiffness and strong energy dissipation of the boundary optimized damper and the interior optimized damper are validated by the simulated quasistatic test, but the integrated performance is necessary to be verified if the two types of dampers are used in the engineering structures. In this following study, the finite element software ANSYS is used for simulation analysis, and the simulation models are established by parametric language APDL. The combined damper is installed on a steel frame with one story and one span, as shown in Figure 22.

The height of the frame is $1.5 \mathrm{~m}$ and the span is $3.0 \mathrm{~m}$. The dimension of the column section is $200 \mathrm{~mm} \times 200 \mathrm{~mm}$, the dimension of the beam section is $200 \mathrm{~mm} \times 180 \mathrm{~mm}$, and the dimension of the diagonal brace is $100 \mathrm{~mm} \times 200 \mathrm{~mm}$. The dimensions of the dampers are the same as sizes shown in Figure 12, and the compositions of the combined dampers are alternatively assembled by 3 pieces of Q345 plates and 2 pieces of LY160 plates. In order to ensure the accuracy of analysis and simplify the analysis process, the beam, the columns, and the dampers are modeled by solid element, and 


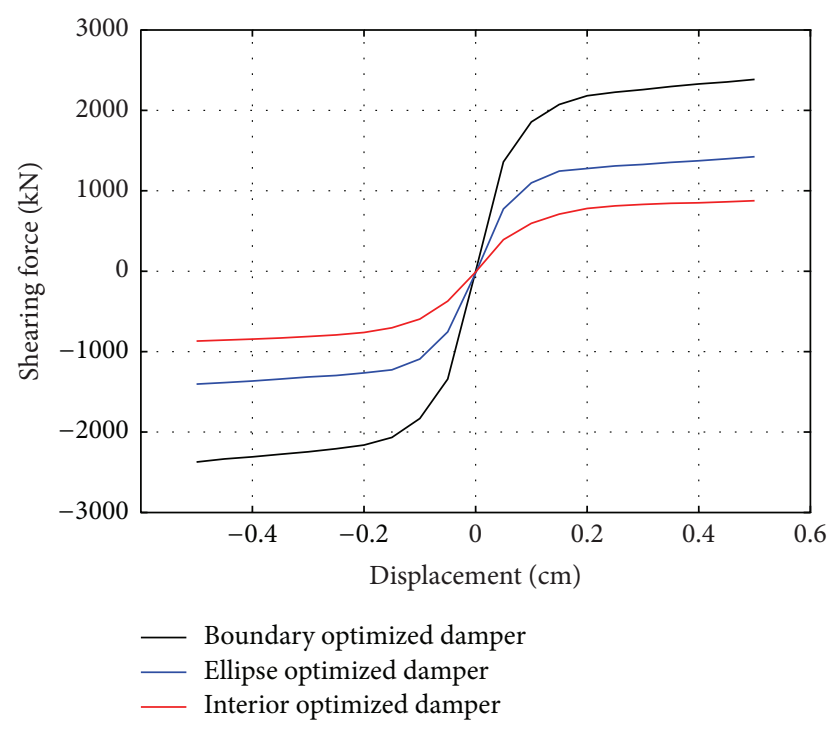

FIGURE 17: Skeleton curves of different dampers.

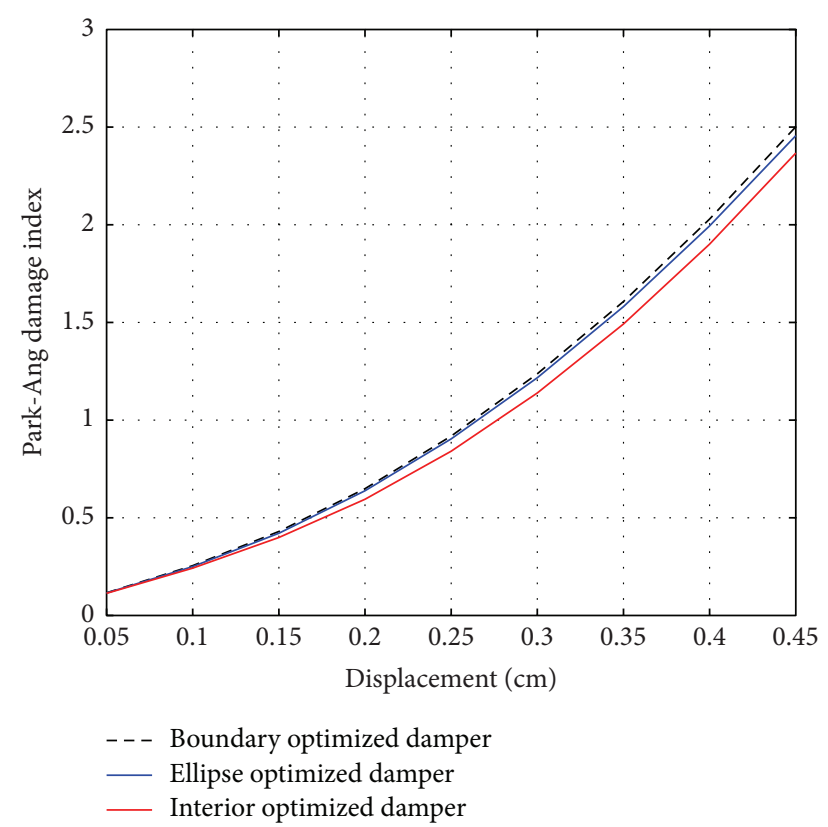

FIgURE 18: Comparison of damage index.

the diagonal braces are simulated by beam element. In the process of model building, the column bottoms have fixed constraints and the cyclic displacement loading is applied on the column top, as shown in Figure 12. The detailed models of the dampers in the frame and the connection connected with the beam and the damper are shown in Figure 23.

The displacement response of the damper under each loading step is extracted and the displacement history is obtained, as shown in Figure 24. The displacements of the two types of dampers are equal in the loading steps 0130 because the structures are still in the elastic state and the equivalent stiffness of the dampers is identical. After

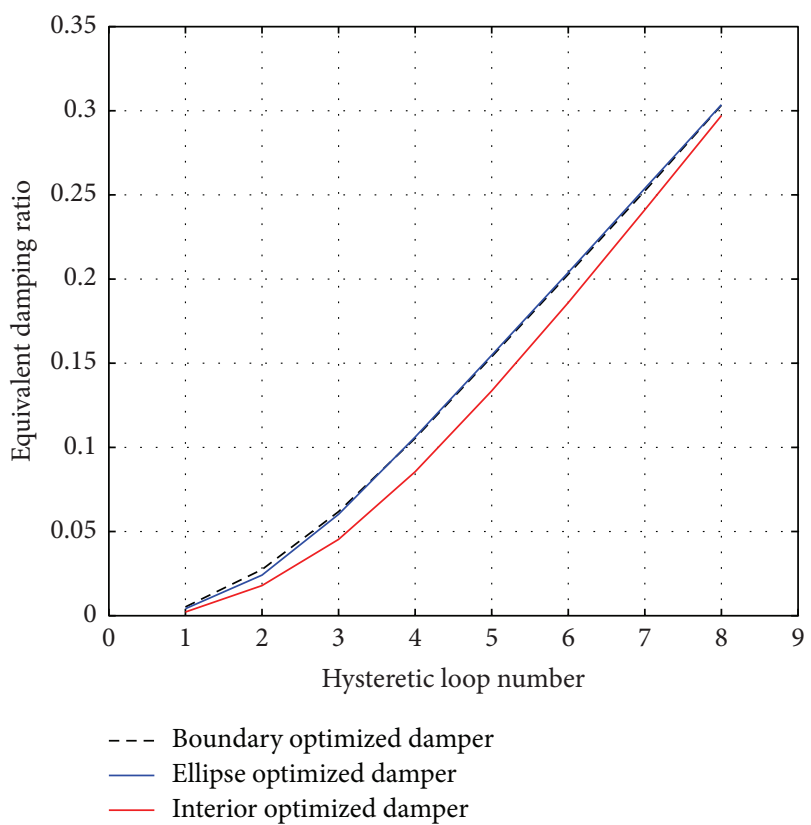

FIGURE 19: Comparison of equivalent damping ratio.

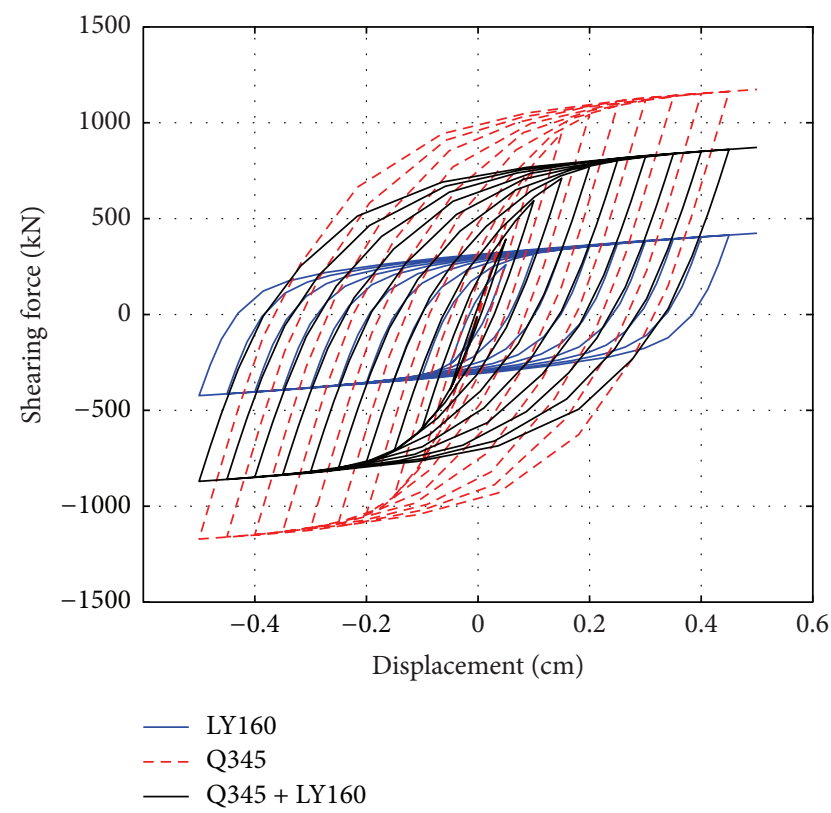

Figure 20: Hysteresis curves of interior optimized damper.

loading step 130, the boundary optimized damper can reduce the structural displacement more effectively because damping starts to work.

The base shear of the frame structure is extracted and the force history is obtained, as shown in Figure 25. It can be seen that the base shear of the frame structure with boundary optimized damper is significantly greater than the corresponding values of structure with interior optimized damper or 


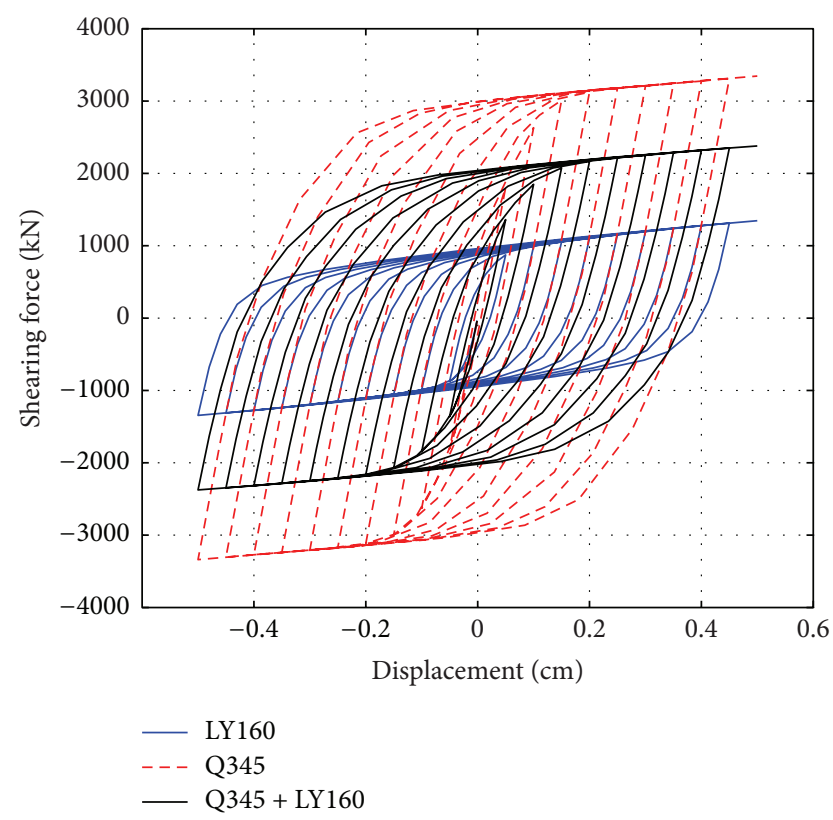

FIGURE 21: Hysteresis curves of boundary optimized damper.

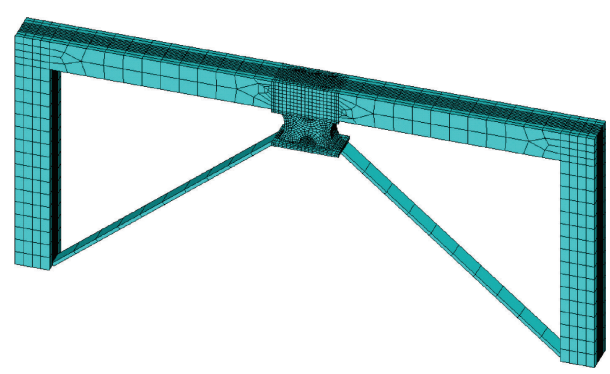

FIGURE 22: Damper model in the frame.

the uncontrolled structure, which indicates that the boundary optimized damper has better energy-dissipation capacity and obviously improves the structural resistance.

The strain nephogram and hysteretic curves of the dampers are shown in Figures 26 and 27, respectively.

It indicates that the nephogram color of the boundary optimized damper is homogeneous and the hysteresis curve is fuller, and the effect of energy dissipation is obvious. The comparison of dissipated energy of each hysteretic loop is shown in Figure 28, and the actual dissipated energy of the boundary optimized damper and the interior optimized damper is $1.349 \times 10^{10} \mathrm{~N} \cdot \mathrm{m}$ and $9.383 \times 10^{9} \mathrm{~N} \cdot \mathrm{m}$, respectively. All the results indicate that the energy-dissipation capacity of the boundary optimized damper is more prominent. Hence, the combined low yield point steel plate damper with boundary optimized shape is recommended in practical engineering applications.

It indicates that the nephogram color of the boundary optimized damper is homogeneous and the hysteresis curve is fuller, and the effect of energy dissipation is obvious. The comparison of dissipated energy of each hysteretic loop is shown in Figure 28, and the actual dissipated energy of the boundary optimized damper and the interior optimized damper is $1.349 \times 10^{10} \mathrm{~N} \cdot \mathrm{m}$ and $9.383 \times 10^{9} \mathrm{~N} \cdot \mathrm{m}$, respectively. All the results indicate that the energy-dissipation capacity of the boundary optimized damper is more prominent.

Though it is verified that the optimized dampers have the superior performances such as large initial stiffness and strong energy-dissipation capacity by quasistatic simulation, the integrated performance is still necessary to be verified if the two types of dampers are used in multilayer frame structures which are subjected to earthquakes. Since the computational efficiency of ANSYS for nonlinear time-history analysis is lower, finite element analysis software ETABS is used to simulate the structural nonlinear responses under earthquake. At first, the same one-layer frame as the previous model of ANSYS is established by ETABS, and the damper element is adopted as the brace connection in the model. By appropriately setting the propertyparameters of the connection element, the hysteresis curve of the connection element under low cyclic loading is close to that of ANSYS, and the results are shown in Figure 29, so the damping parameter in ETABS is assumed to be accurate. For the final optimal results, the damping exponent of the edge optimized damper is 2.00 , and the damping is $3000 \mathrm{kN} \cdot \mathrm{s} / \mathrm{m}$. The damping exponent of the interior optimized damper is 1.85 , and the damping is $3500 \mathrm{kN} \cdot \mathrm{s} / \mathrm{m}$.

In order to study the damping performance of the multilayer structure with optimized dampers, a one-dimensional and three-layer steel frame with boundary optimized dampers or interior optimized dampers is established by ETABS, respectively. Besides, the uncontrolled frame model is established as a reference structure, and there are no dampers but only the same braces on it. The corresponding plane graphs of the frame structure are shown in Figure 30. The height of the steel frame is $3.6 \mathrm{~m}$, and the span is $6.0 \mathrm{~m}$. The frame column cross section is $\mathrm{H} 400 \times 300 \times 25 \times 25(\mathrm{~mm})$ and the beam section size is $\mathrm{H} 250 \times 150 \times 20 \times 12(\mathrm{~mm})$. The diagonal brace section size is $\mathrm{H} 150 \times 150 \times 12 \times 8(\mathrm{~mm})$. The yield strength of all the components is $235 \mathrm{MPa}$. The dampers are simulated according to the previous parameters. The site type is soft rock and the seismic fortification intensity is 8.0. The fundamental period of the uncontrolled frame and controlled frame is $0.194 \mathrm{~s}$ and $0.197 \mathrm{~s}$, respectively.

In order to study and compare the seismic performance of these three kinds of structures, a series of typical onedimensional earthquake waves which include El Centro earthquake, Taft earthquake, and Michoacán earthquake are selected because these ground motion records were collected from hard soil and the predominant periods are close to the structural period; then nonlinear time-history analysis are carried out. To consider the effect of ground motion amplitude on the damage degree, the peak ground motion in the horizontal direction is separately adjusted to $0.11 \mathrm{~m} / \mathrm{s}^{2}$, $0.22 \mathrm{~m} / \mathrm{s}^{2}$, and $0.51 \mathrm{~m} / \mathrm{s}^{2}$, so as to adjust the earthquake waves as the excitation for small earthquake, moderate earthquake, and severe earthquake, respectively. 


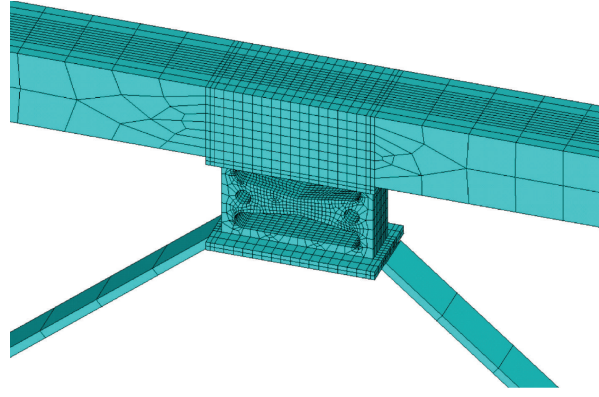

(a) Interior optimized damper

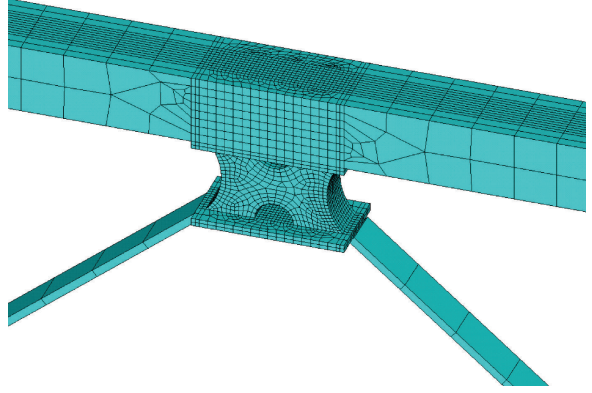

(b) Boundary optimized damper

Figure 23: Optimized damper connected with beam.

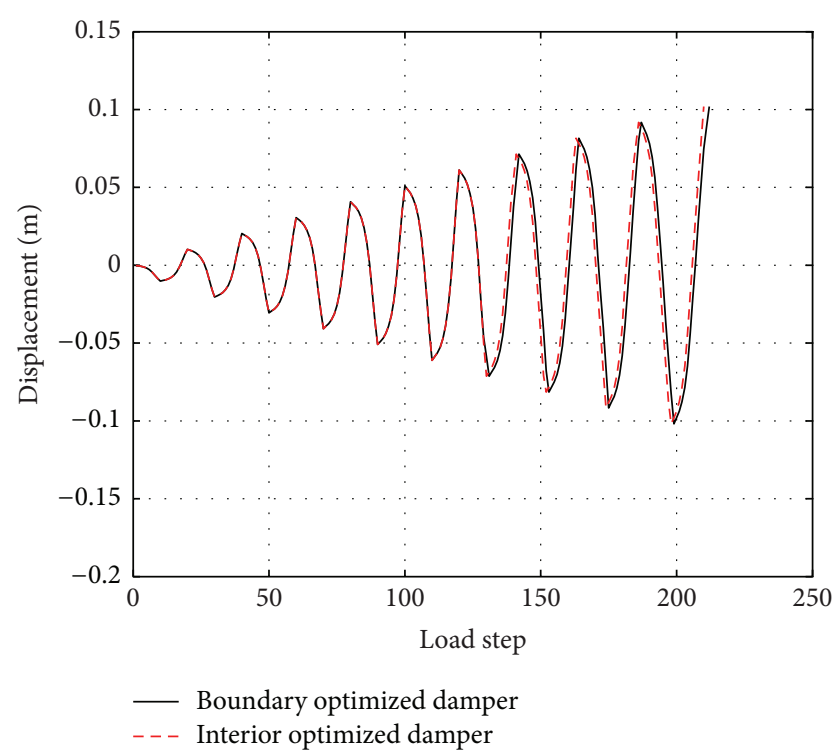

FIGURE 24: Comparison of displacement history in frame.

The top displacements and the top accelerations of various structures which are subjected to earthquakes with different amplitudes are extracted and analyzed. The specific results are shown in Table 2. In this study, the peak damping ratio (PDR) refers to the ratio between the differences of the absolute maximum responses before and after vibration control and the absolute maximum structural response before control. The envelope damping ratio (EDR) refers to the ratio between the differences of the sum of absolute responses before and after vibration control and the sum of absolute response before control. It should be noted that plan A means the structure with edge optimized dampers and plan B means the structure with interior optimized dampers. In addition, the top displacement history curves of various structures which are subjected to El Centro wave with different amplitudes are shown in Figures 31-33.

It can be seen from Table 2 and Figures 31-33 that the damping effects of the edge optimized damper and

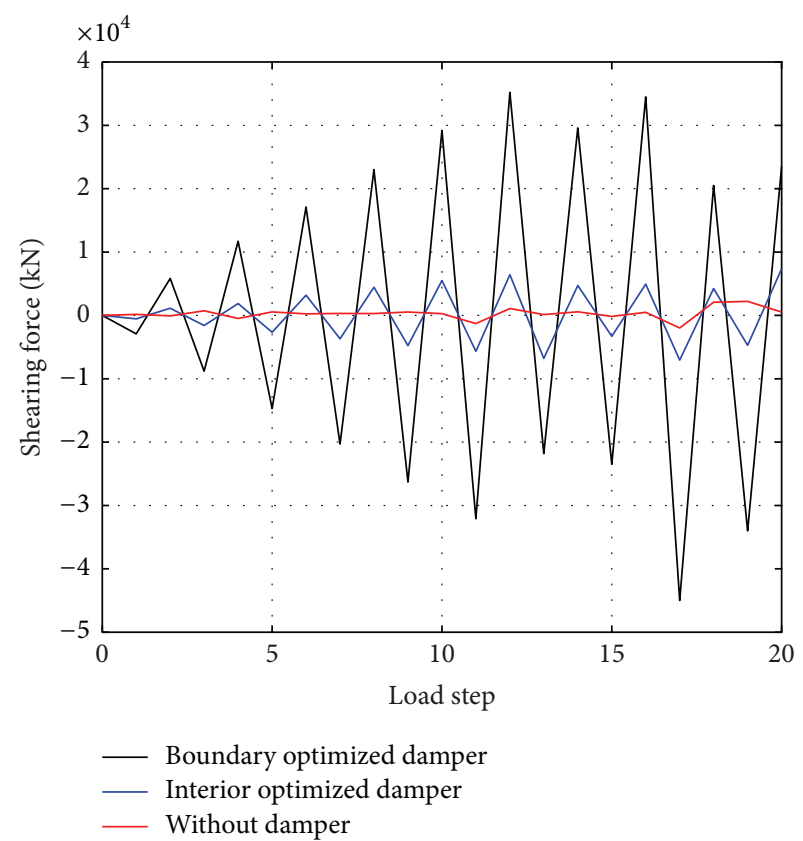

FIGURE 25: Comparison of force history.

the interior optimized damper are both superior, and the former has a stronger damping capacity. Furthermore, the inelastic energy-dissipation capacity of the damper is gradually highlighted with the increase of peak ground acceleration, and the damping effect is more obvious. In summary, the damping performance of the low yield point metal damper is excellent and stable, and it can significantly improve the structural seismic capacity. In practical engineering applications, the boundary optimized damper is still the preferred option.

In view of the adoption for practical applications, a design procedure of the combined low yield point steel plate damper is finally proposed based on the above analysis. According to the concept of performance based seismic 
TABLE 2: Results of damping effect.

\begin{tabular}{|c|c|c|c|c|c|c|c|c|}
\hline \multirow{3}{*}{ Earthquake } & \multicolumn{8}{|c|}{ Damping effect } \\
\hline & \multicolumn{2}{|c|}{ PDR of top displacement (\%) } & \multicolumn{2}{|c|}{ PDR of top acceleration (\%) } & \multicolumn{2}{|c|}{ EDR of top displacement (\%) } & \multicolumn{2}{|c|}{ EDR of top acceleration (\%) } \\
\hline & Plan A & Plan B & Plan A & Plan B & Plan A & Plan B & Plan A & Plan B \\
\hline \multicolumn{9}{|l|}{ El Centro } \\
\hline Small & 23.50 & 13.83 & 55.17 & 42.39 & 20.69 & 14.99 & 81.30 & 76.57 \\
\hline Medium & 29.43 & 21.31 & 61.50 & 49.93 & 30.76 & 21.82 & 85.31 & 81.19 \\
\hline Large & 66.88 & 48.98 & 67.23 & 58.13 & 62.53 & 42.48 & 88.29 & 84.90 \\
\hline \multicolumn{9}{|l|}{ Taft } \\
\hline Small & 49.37 & 32.74 & 69.64 & 68.78 & 52.34 & 47.30 & 71.00 & 65.07 \\
\hline Medium & 49.56 & 43.04 & 84.78 & 74.76 & 54.46 & 51.61 & 87.67 & 80.71 \\
\hline Large & 74.63 & 60.68 & 89.96 & 82.26 & 78.14 & 66.64 & 91.84 & 85.60 \\
\hline \multicolumn{9}{|l|}{ Michoacán } \\
\hline Small & 68.29 & 64.63 & 61.18 & 51.29 & 60.52 & 55.82 & 78.10 & 73.24 \\
\hline Medium & 74.39 & 70.43 & 66.87 & 58.19 & 68.74 & 64.67 & 83.11 & 76.91 \\
\hline Large & 79.95 & 75.23 & 80.74 & 61.49 & 77.21 & 65.09 & 91.23 & 84.46 \\
\hline
\end{tabular}

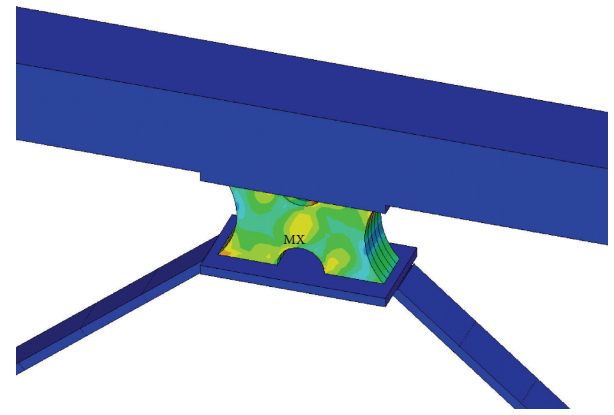

FIGURE 26: Strain nephogram of boundary optimized damper.

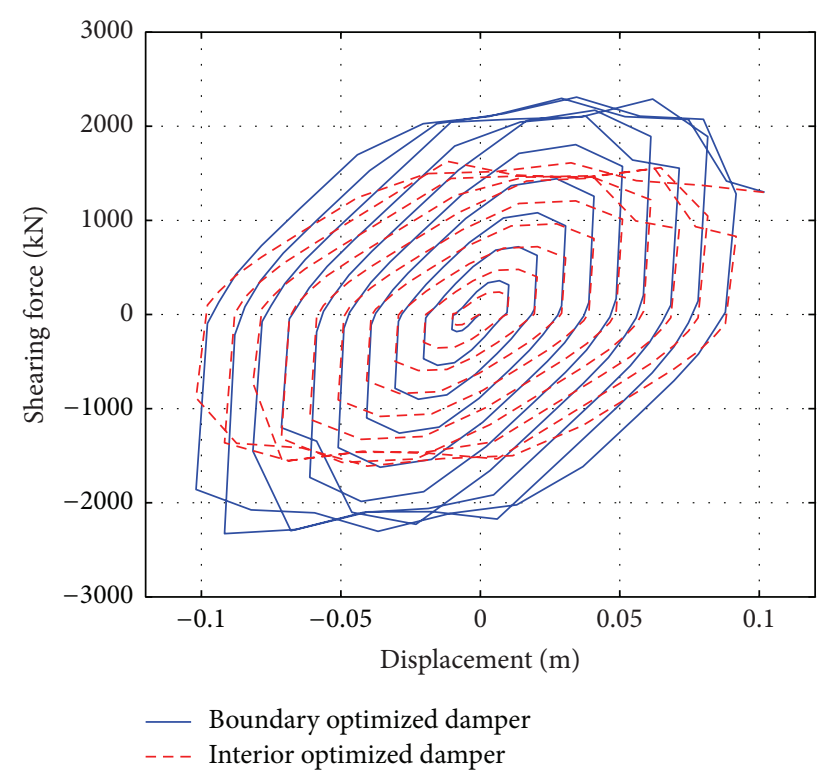

FIGURE 27: Hysteretic curves of damper.

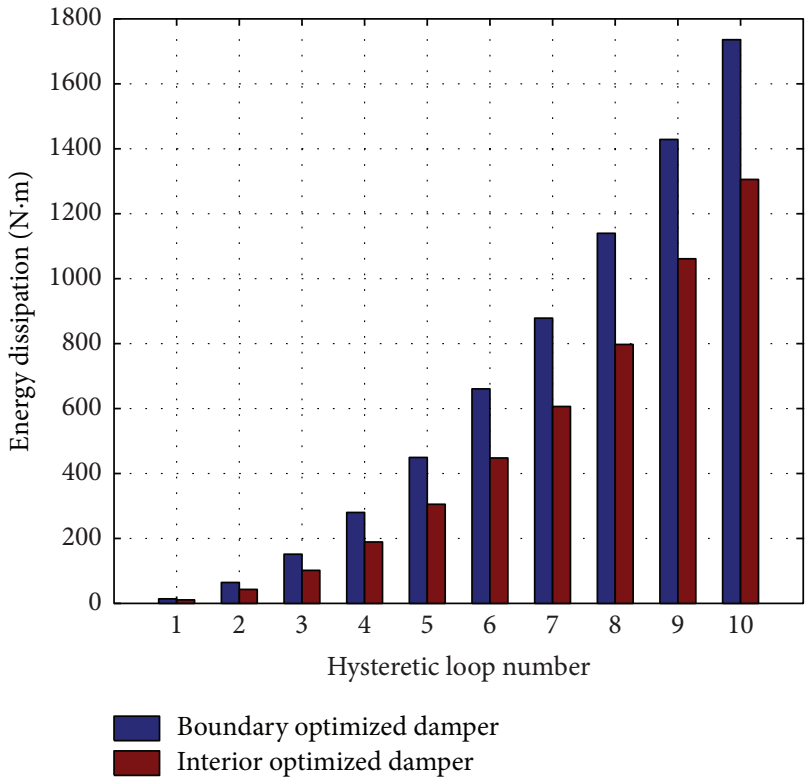

FIGURE 28: Comparison of energy dissipation of different dampers.

design, a generalized displacement-based design procedure of hysteretic damped braces has been proposed by Mazza and Vulcano [28, 29], which proportions hysteretic damped braces to attain a designated performance level of an existing framed structure. On the basis of this research, the main steps of the proposed design procedure of frame with combined steel plate damper are summarized below:

(1) Carry out pushover analysis of the unbraced frame and definition of an equivalent single degree of freedom system to evaluate the equivalent viscous damping due to hysteresis loading. 


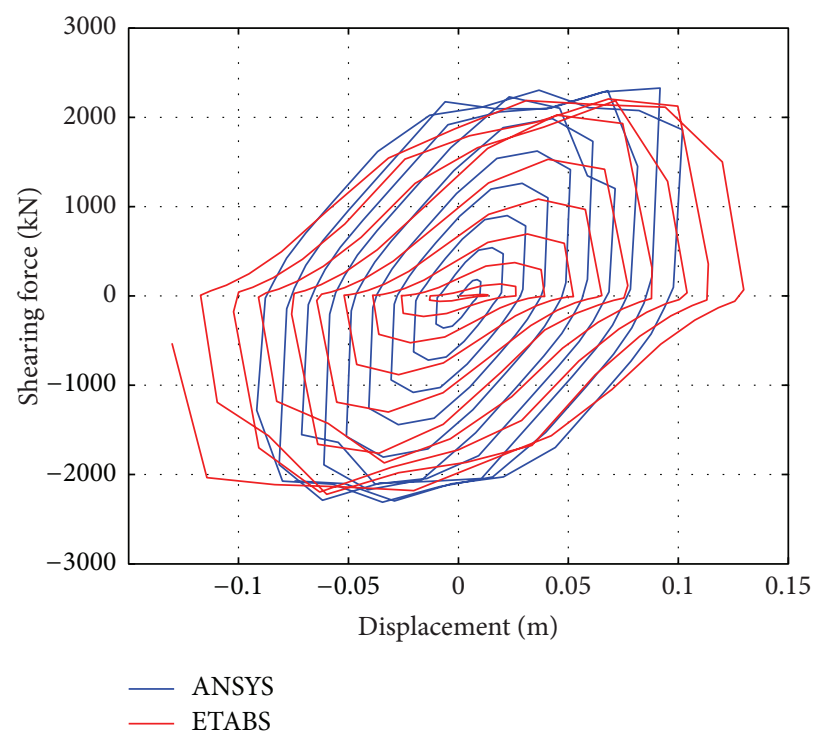

(a) Boundary optimized damper

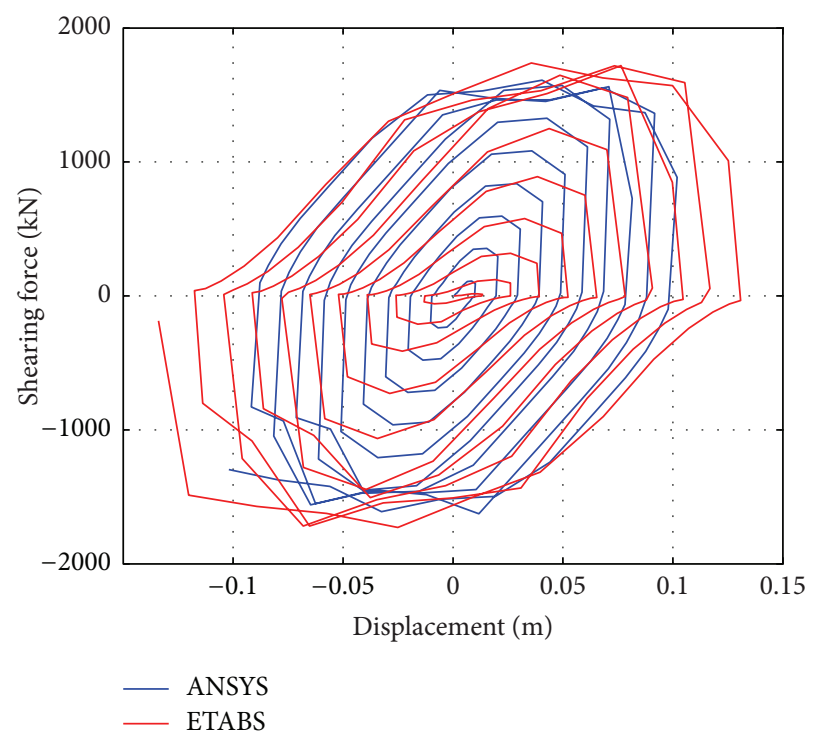

(b) Interior optimized damper

Figure 29: Comparison of hysteresis curves using ANSYS and ETABS.

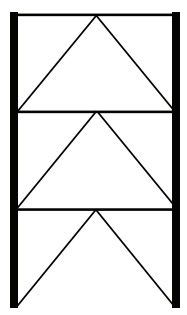

(a) Frame without dampers

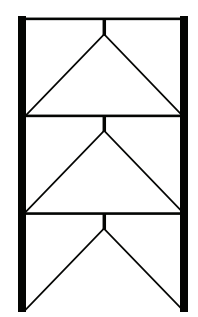

(b) Frame with dampers

FIGURE 30: Plane graph of uncontrolled frame and controlled frame.

(2) Determine the equivalent viscous damping due to hysteresis loading of the damped braces according to the integral ductility demand.

(3) Determine the shape of the dampers based on topology optimization, and both "maximum stiffness" and "full stress state" are used as the optimization objectives.

(4) Determine the equivalent viscous damping of the frame with damped braces.

(5) Determine the effective stiffness of the equivalent damped brace based on effective period.

(6) Determine the effective strength properties of the equivalent damped brace based on effective yield base shear.

(7) Carry out design of the hysteretic damped braces of the controlled frame considering special demands.

\section{Conclusion}

In order to solve the deficiencies such as high yield point and low adjustability of the traditional low yield point plate steel damper, three kinds of combined low yield point plate steel dampers which consist of low yield point steel plates and common steel plates in different proportions are proposed. Based on the concept of "maximum stiffness" and "full stress state," the dampers with interior hollows, boundary hollows, and ellipse hollows are optimized by alternating topology optimization method, and three types of combined low yield point steel plate dampers are obtained, which at the same time meet the optimization objectives "maximum stiffness" and "full stress state."

Finite element models of the optimized various combined steel plate dampers are analyzed. The results show that the interior optimized damper is suitable for the structure with large deformation, and the energy-dissipation capacity of the boundary optimized damper is excellent and 


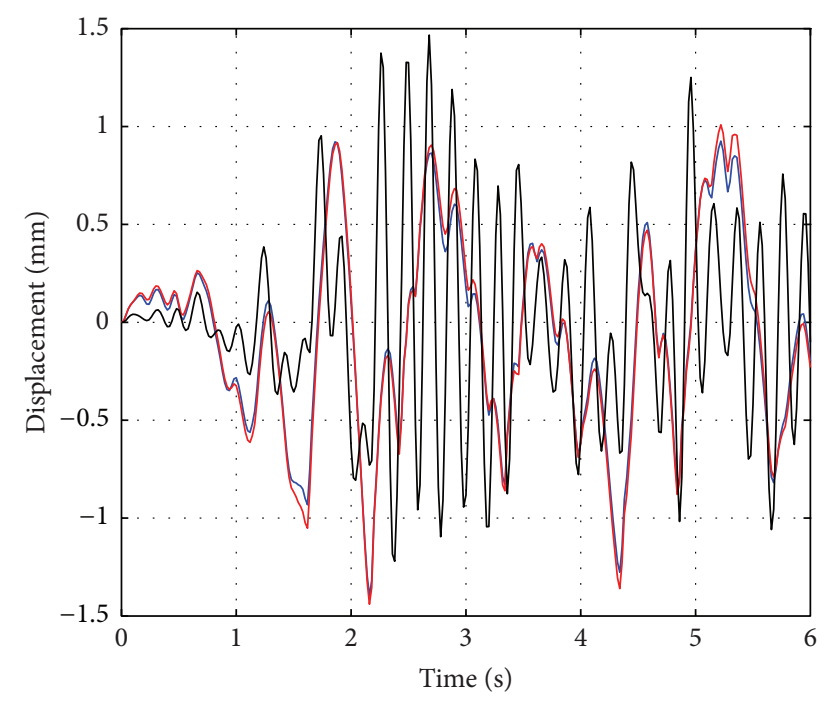

With boundary optimized damper
With interior optimized damper
Uncontrolled frame

FIgURE 31: Time-history curve under small earthquake.

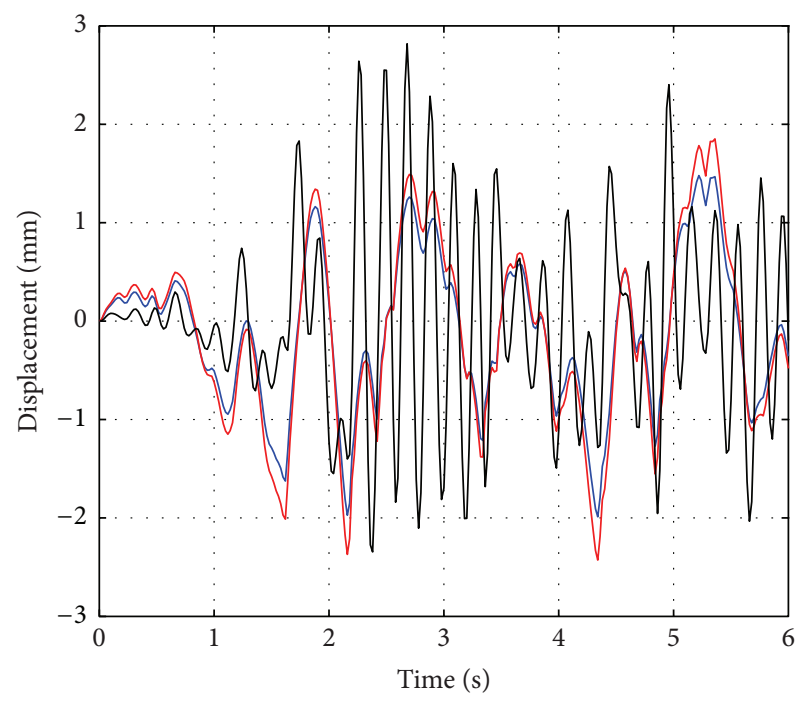

_ With boundary optimized damper
_ With interior optimized damper

FIgURE 32: Time-history curve under medium earthquake.

the comprehensive performance is optimal. Two kinds of optimization models with different material ratios are studied by numerical simulation, and it is verified that the yield stress of combined dampers can be regulated through changing material proportion.

In order to verify the actual damping effect on the structure installed combined dampers, the seismic capacity of a steel frame structure with different types of dampers is studied by simulation analysis. The results indicate that the boundary optimized damper has better energy-dissipation

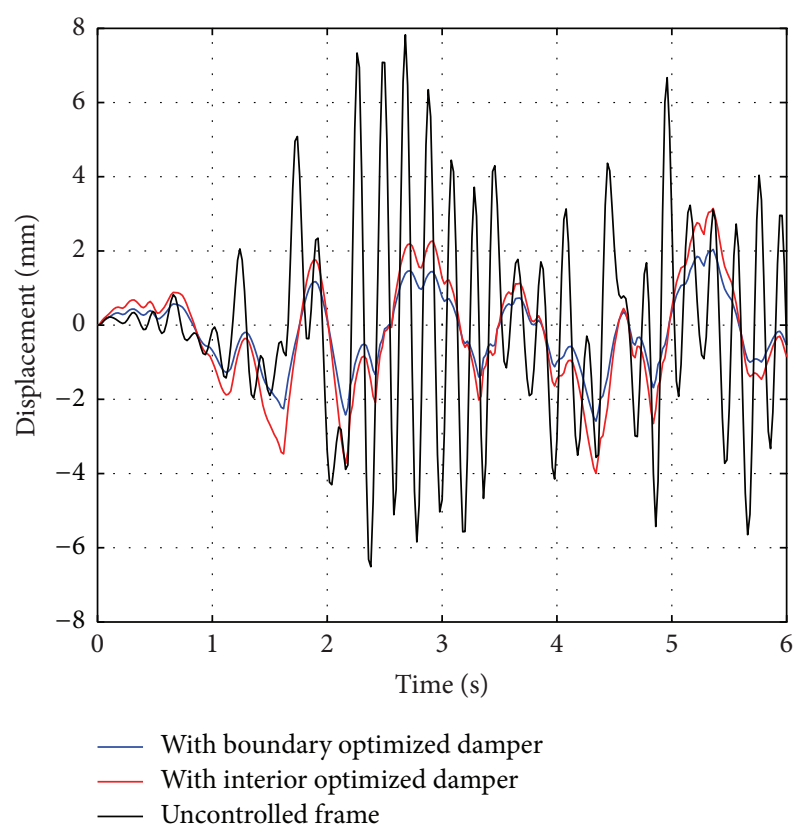

FIGURE 33: Time-history curve under large earthquake.

capacity than the interior optimized damper. In practical engineering applications, the boundary optimized damper is recommended.

\section{Competing Interests}

The authors declare that there are no competing interests regarding the publication of this paper.

\section{Acknowledgments}

This work is partially supported by Natural Science Foundation of China under Grants nos. 51478024 and 51108009 and Foundation of Beijing Key Lab of Earthquake Engineering and Structural Retrofit under Grant no. USDE201403.

\section{References}

[1] C. J. Zhang, A. Q. Li, and M. Zhao, "Summary of research on and applications of passive energy dissipation systems of mild steel damper," Industrial Construction, vol. 36, no. 9, pp. 17-21, 2006.

[2] J. M. Kelly, R. I. Skinner, and A. J. Heine, "Mechanisms of energy absorption in special devices for use in earthquake-resistant structures," Bulletin of the New Zealand Society for Earthquake Engineering, vol. 5, no. 3, pp. 63-88, 1972.

[3] A. S. Whittaker, V. V. Bertero, C. L. Thompson, and L. J. Alonso, "Seismic testing of steel plate energy dissipation devices," Earthquake Spectra, vol. 7, no. 4, pp. 563-604, 1991.

[4] K.-C. Tsai, H.-W. Chen, C.-P. Hong, and Y.-F. Su, "Design of steel triangular plate energy absorbers for seismic-resistant construction," Earthquake Spectra, vol. 9, no. 3, pp. 505-528, 1993.

[5] L.-D. Tirca, D. Foti, and M. Diaferio, "Response of middle-rise steel frames with and without passive dampers to near-field 
ground motions," Engineering Structures, vol. 25, no. 2, pp. 169$179,2003$.

[6] Y. Zhou and J. Liu, "Experimental study on the behavior of circular ring energy dissipator," World Information on Earthquake Engineering, vol. 11, no. 4, pp. 1-7, 1996.

[7] Y. Zhou and J. Liu, "Experimental study on double circular ring mild steel energy dissipator," Journal of Earthquake Engineering \& Engineering Vibration, vol. 18, no. 2, pp. 117-123, 1998.

[8] W.-Y. Zhang, M.-Z. Zhang, and D.-W. Li, "An experimental research on performance and application of a new type of mild steel damper added damping and stiffness (ADAS)," Journal of Harbin Institute of Technology, vol. 40, no. 12, pp. 1888-1894, 2008.

[9] M. Mito, R. Tamura, and K. Shimizu, "Study on seismic design method for building with steel damper (part1)," in Proceedings of the AIJ Annual Meeting, 1997.

[10] G. Li and H. Li, "Study on vibration reduction of structure with a new type of mild metallic dampers," Journal of Vibration and Shock, vol. 25, no. 3, pp. 66-72, 2006.

[11] J. Tian, Z. C. Yan, and J. L. Lu, "Seismic control research of the multi-ribbed slab structure using the ultra-low yield strength steel," Journal of Vibration and Shock, vol. 33, no. 5, pp. 160-164, 2014.

[12] GB/T 228.1-2010 Metallic Materials Tensile Testing Part 1: Method of Test at Room Temperature, China Standards Press, Beijing, China, 2011.

[13] S.-T. Xing and X. Guo, "Study on mechanical behavior and effectiveness of a new type of mild steel damper," Journal of Earthquake Engineering and Engineering Vibration, vol. 23, no. 6, pp. 179-186, 2003.

[14] K. Deng, P. Pan, J. Sun, J. Liu, and Y. Xue, "Shape optimization design of steel shear panel dampers," Journal of Constructional Steel Research, vol. 99, pp. 187-193, 2014.

[15] Q. Wang and L. G. An, "Analysis on vibration suppression of a new type of planar steel damper," China Civil Engineering Journal, vol. 43, pp. 341-344, 2010.

[16] Y. Liu and M. Shimoda, "Shape optimization of shear panel damper for improving the deformation ability under cyclic loading," Structural and Multidisciplinary Optimization, vol. 48, no. 2, pp. 427-435, 2013.

[17] M. Ohsaki, Optimization of Finite Dimensional Structures, CRC Press, 2010.

[18] P. Pan, M. Ohsaki, and H. Tagawa, "Shape optimization of Hbeam flange for maximum plastic energy dissipation," Journal of Structural Engineering, vol. 133, no. 8, pp. 1176-1179, 2007.

[19] G. N. Vanderplaats, "Multidiscipline design optimization," Applied Mechanics Reviews, vol. 41, no. 6, pp. 257-262, 1988.

[20] U. Kirsch, M. Bogomolni, and I. Sheinman, "Efficient procedures for repeated calculations of the structural response using combined approximations," Structural and Multidisciplinary Optimization, vol. 32, no. 6, pp. 435-446, 2006.

[21] Y. Liu and M. Shimoda, "Shape optimization of shear panel damper for improving the deformation ability under cyclic loading," Structural \& Multidisciplinary Optimization, vol. 48, no. 2, pp. 427-435, 2013.

[22] X. Huang and Y. M. Xie, "A new look at ESO and BESO optimization methods," Structural \& Multidisciplinary Optimization, vol. 35, no. 1, pp. 89-92, 2008.

[23] Q. M. Liu, "A design method of rib distribution for thin plate structure based on the full stress rule," Machinery Design \& Manufacture, vol. 1, no. 1, pp. 23-24, 2007.
[24] X. H. Ding and L. Cheng, "Topology optimization of fullstressed structures based on SKO method," China Mechanical Engineering, vol. 20, no. 15, pp. 1765-1770, 2009.

[25] Y.-J. Park, A. H.-S. Ang, and Y. K. Wen, "Seismic damage analysis of reinforced concrete buildings," Journal of Structural Engineering, vol. 111, no. 4, pp. 740-757, 1985.

[26] FEMA274, NEHRP Commentary on the Guidelines for the Seismic Rehabilitation of Buildings, Federal Emergency Management Agency, Washington, DC, USA, 1997.

[27] ATC40, Seismic Evaluation and Retrofit of Concrete Buildings, ATC, Redwood City, Calif, USA, 1996.

[28] F. Mazza, "Nonlinear seismic analysis of unsymmetric-plan structures retrofitted by hysteretic damped braces," Bulletin of Earthquake Engineering, vol. 14, no. 6, pp. 1311-1331, 2016.

[29] F. Mazza, M. Mazza, and A. Vulcano, "Displacement-based seismic design of hysteretic damped braces for retrofitting inelevation irregular r.c. framed structures," Soil Dynamics and Earthquake Engineering, vol. 69, no. 2, pp. 115-124, 2015. 

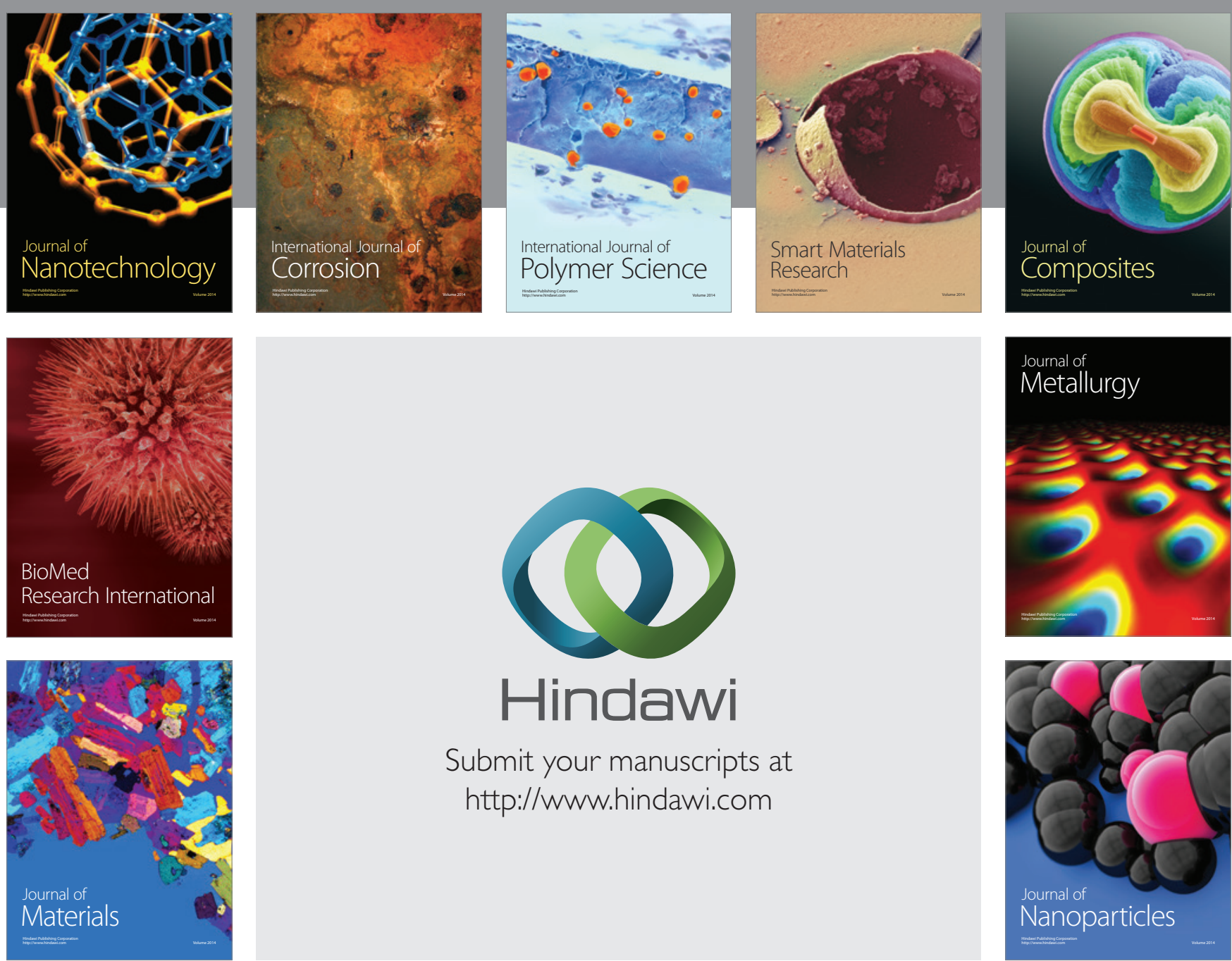

\section{Hindawi}

Submit your manuscripts at

http://www.hindawi.com

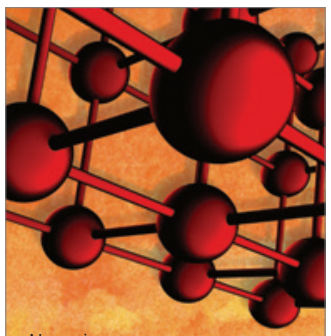

Materials Science and Engineering
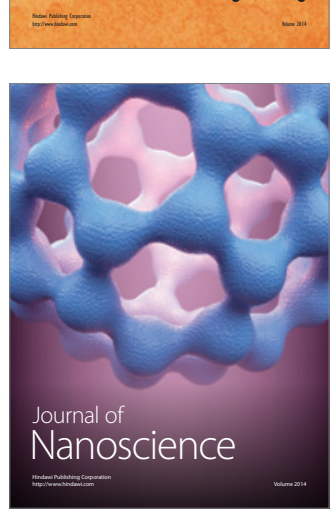
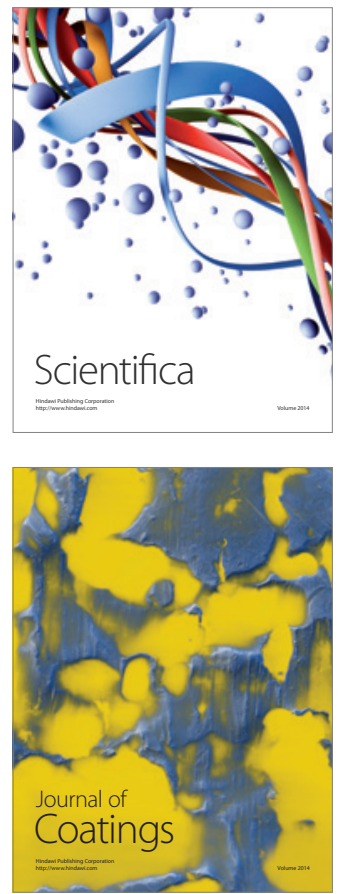
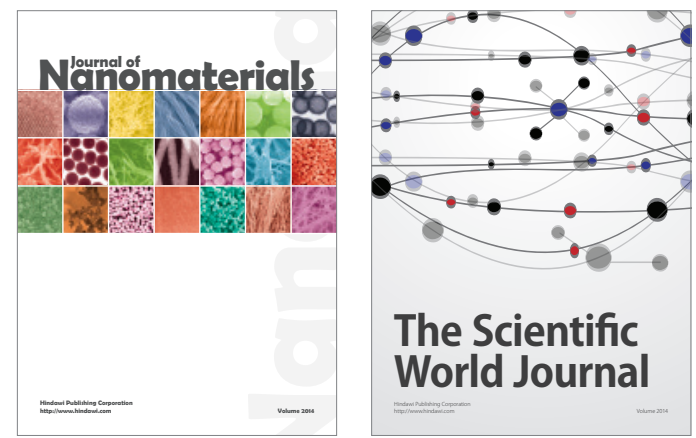

The Scientific World Journal
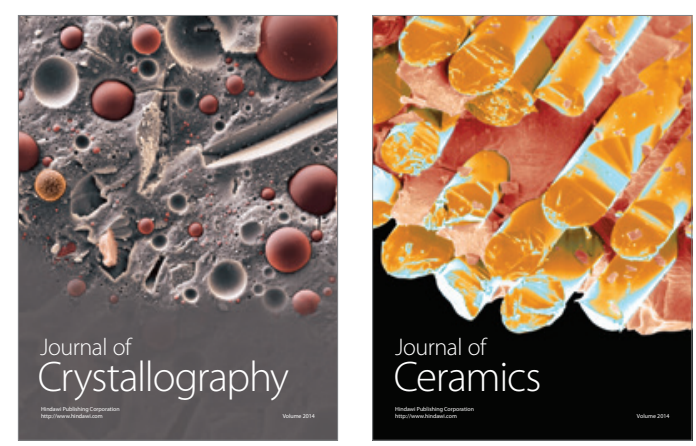
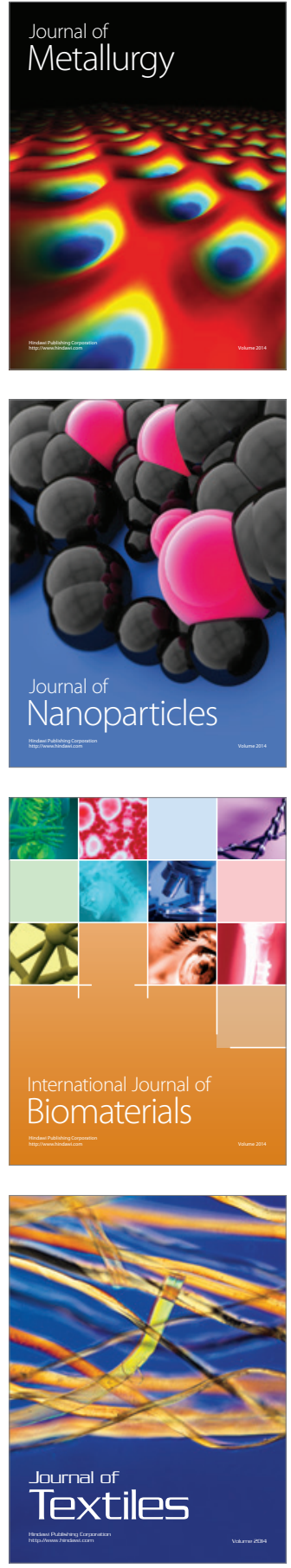